INT. J. REMOTE SENSING, 1990, vOL. 11, NO. 10, 1695-1727

\title{
Monte-Carlo simulation studies of the nonlinear imaging of a two dimensional surface wave field by a synthetic aperture radar
}

\author{
CLAUS BRÜNING \\ Max-Planck-Institut für Meteorologie, Bundesstraße 55, 2000 Hamburg 13, \\ F.R. Germany \\ WERNER ALPERS \\ Universität Hamburg, Institut für Meereskunde, Troplowitzstraße 7, \\ 2000 Hamburg 54, F.R. Germany \\ and KLAUS HASSELMANN \\ Max-Planck-Institut für Meteorologie, Bundesstraße 55, 2000 Hamburg 13, \\ F.R. Germany
}

(Received 4 May 1988; in final form 28 June 1989)

\begin{abstract}
The imaging of ocean surface waves by synthetic aperture radar (SAR) is investigated using two-dimensional Monte-Carlo simulations. The properties of the SAR imaging mechanism for windseas and swell in the Bragg scattering regime are discussed as a function of a few governing non-dimensional parameters formed from a combination of SAR and ocean wave parameters. The parameter ranges may be classified into three regimes corresponding to linear and weakly nonlinear, medium nonlinear and strongly nonlinear imaging. The nonlinearities are induced by motion effects (velocity bunching, velocity spread and acceleration smearing), while the real aperture radar (RAR) tilt and hydrodynamic modulation processes are regarded as linear. In the strongly nonlinear imaging regime, the velocity bunching mechanism causes a rotation of the spectral peak towards the range direction and a stretching of the peak wavelength. In addition, the azimuthal resolution is degraded through the Doppler spreading arising from the different facet velocities within a SAR resolution cell. The imaging properties in this regime are largely governed by two non-dimensional parameters, the velocity bunching and velocity smearing parameter. The nonlinear imaging distortions are strongest for broad spectra (windseas) and are significantly weaker for narrow-band swell. In the linear and weakly nonlinear imaging regime, the superposition of the hydrodynamic and tilt cross-section modulation and the velocity bunching transfer function normally produces a rotation of the spectral peak towards the azimuthal direction. The interference characteristics of these different modulation mechanisms depends on the wave propagation direction and can lead to a significant distortion of the image. This is often seen in large differences in the image modulation depths of waves propagating parallel and anti-parallel to the flight direction.
\end{abstract}

\section{Introduction}

The imaging of the ocean surface by synthetic aperture radar (SAR) is known to be strongly influenced by the orbital motions of the surface waves. The Doppler shifts induced by these motions distort the phase history of the backscattered signal which is used to synthesize the azimuthal resolution. Their effect on the SAR imaging has been studied in many investigations. However, most explicit numerical calculations have been restricted either to the linear mapping regime, for which the linear superposition 
principle applies, or to single sinusoidal waves (see e.g. Elachi and Brown 1977, Alpers and Rufenach 1979, Swift and Wilson 1979, Valenzuela 1980, Raney 1981, Rufenach and Alpers 1981, Rotheram 1983, Plant and Keller 1983, Ivanov 1983, Ouchi 1984, Tucker 1985, Hasselmann et al. 1985). Unfortunately, the SAR imaging mechanism of ocean waves is often nonlinear, so that the SAR imaging of a continuous wave spectrum cannot be described generally by the superposition of linearly mapped sinusoids. Similarly, it is not clear how the nonlinear mapping computations for a single sinusoid can be carried over into the realistic case of an arbitrary spectral continuum.

In this paper we study the nonlinear imaging of a two dimensional random surface wave field by means of Monte-Carlo mapping computations. This method was first applied by Alpers (1983) to the imaging of one-dimensional ocean wave fields. His simulations, however, although giving valuable insight into several features of the SAR imaging mechanism, were unable to model many important properties of the two-dimensional mapping process adequately. This includes, in particular, the most striking characteristics of SAR wave images: the pronounced asymmetries observed between the imaging of range and azimuthally travelling waves.

Two-dimensional Monte-Carlo simulation studies have been applied previously in a comparison study of ocean wave spectra measured with a buoy and SAR image spectra obtained over the North Sea during the Shuttle Imaging Radar-B (SIR-B) mission (Alpers et al. 1986). Similar two-dimensional Monte-Carlo simulations have been made by Alpers and Brüning (1986) in a pilot study to assess the relative importance of the various motion-induced contributions to the SAR imaging mechanism of ocean waves and also by Lyzenga (1986).

Since the work described in this paper has been completed, a new closed integral relation for the nonlinear transformation of an ocean wave spectrum to a SAR image spectrum has been derived by Hasselmann and Hasselmann (1990). The transformation computations using this expression are significantly faster than Monte-Carlo computations and avoid the statistical sampling errors of the latter approach. The closed transformation expression can also be readily inverted using standard inverse modelling methods. The new relation has been applied to studies of LEWEX wave and SAR spectra (Hasselmann et al. 1990).

In the present paper, we attempt a more complete investigation of the SAR imaging mechanism using the Monte-Carlo technique in which the parameter ranges for the linear and nonlinear imaging regimes are systematically explored. The motivation of this study is to provide a computational data base in support of future inversion algorithms for extracting information on ocean wave spectra from twodimensional SAR image spectra in future satellite and Space Shuttle missions such as ERS-I, Radarsat and SIR-C.

\section{The ocean wave SAR imaging model}

The SAR imaging model used in this investigation is the generalized velocity bunching model described in Alpers and Rufenach (1979), Alpers et al. (1981), Alpers (1983), Hasselmann et al. (1985), and Alpers and Brüning (1986). The electromagnetic interaction of the SAR microwave radiation with the ocean wave field is described by a two-scale wave model (Wright 1968, Bass et al. 1968) in which the complex reflectivities (backscattering coefficients) of spatially separated backscattering elements (facets) are assumed to be uncorrelated in phase, but to be modulated in amplitude by the long waves. In the basic backscattering two-scale model the 
separation scale is defined as the facet dimension. In applying the model to a SAR it is useful to introduce also an additional separation scale given by the SAR resolution scale. This divides the ocean wave field into deterministic and statistical regimes. Ocean waves longer than the SAR resolution scale can be imaged explicitly by the SAR while the subresolution scale waves need to be described statistically. In general, the SAR resolution scale is an order of magnitude larger than the facet dimension.

It is normally assumed that the backscattering mechanism is primarily Bragg scattering but this is not essential for the formulation of the general theory (an application of the theory to the specular reflection regime is given in Winebrenner and Hasselmann 1988). A detailed discussion of the validity of the assumptions entering into this model is given in Hasselmann et al. (1985). In the present application, Bragg backscattering will be assumed, since ocean wave imaging SARs normally operate in the range of incidence angles between $20^{\circ}$ and $60^{\circ}$ in which this is the dominant imaging mechanism.

The physical processes responsible for the imaging of surface waves consist of the tilt and hydrodynamic cross-section modulation, the so-called RAR (real aperture radar) or amplitude modulation processes, and the motion effects, or the phase modulation processes.

Tilt modulation is a purely geometrical effect. It describes the variations in backscattered energy due to changes in the local radar incidence angles as the directions of the facet normals are modulated along the long wave profile. The hydrodynamic modulation arises from the interaction between the Bragg scattering waves and the long ocean waves. This produces a non-uniform distribution of the short Bragg waves with respect to the long ocean waves. In the Bragg backscattering regime, the RAR cross-section modulation, defined as the superposition of these two processes, is generally assumed to be linearly dependent on the long ocean wave field.

The motion effects influence the SAR imaging mechanism by distorting the complex reflectivity, in particular the phase history of the signal returned from a backscattering element (facet), which is used by the SAR to reconstruct the azimuthal coordinates of the individual scattering elements. The phase variation of a backscattering facet is determined by the radial component (i.e., the component directed towards the radar) of the orbital motions of the long ocean waves. It can be characterized, for the finite time in which the scattering element is viewed by the SAR, by a first order mean Doppler offset proportional to the mean radial orbital velocity and a second order Doppler broadening proportional to the mean radial orbital acceleration ('mean' refers here to the SAR viewing time).

The Doppler offset gives rise to an azimuthal offset in the image which alternates with the sign of the long orbital velocity. The alternating azimuthal displacements of the facets produce associated variations of the apparent facet density, thereby visualizing the waves even when no cross-section modulation is present. However, when this 'velocity bunching', mechanism becomes so strong that the facet displacements become comparable with or larger than the long wave wavelengths, the wave patterns in the SAR image become severely distorted or can even be completely smeared out.

The Doppler broadening of a backscattering facet arises from the quadratic phase error induced by the radial orbital acceleration of the long ocean waves. This causes a (spatially variable) azimuthal smearing of the image, i.e. a degradation of the azimuthal resolution. In the terminology of the SAR two-scale model, 'long waves' refer here to all waves whose half wavelength exceeds the SAR resolution scale. 
In addition to these imaging properties of individual backscattering facets, a further azimuthal smearing occurs when considering the image of a complete SAR resolution cell. This is caused by the Doppler spreading of the net backscattered return from a resolution cell due to the sub-resolution scale variations of the orbital velocities of different backscattering facets within the cell. The origin of this azimuthal smearing is, of course, still the basic velocity bunching mechanism. In principle, the effect could be described deterministically without recourse to the SAR two-scale concept, if the SAR imaging process were simulated in our Monte-Carlo computation with a sufficiently high resolution of the sea surface of the order of the facet dimension. But it is more efficient, and entirely equivalent, to choose the horizontal sea surface resolution equal to the SAR resolution scale, and to describe the contribution of the subresolution scale velocity bunching equivalently as a statistical velocity spread term.

In contrast to the acceleration smearing, the velocity spread azimuthal smearing is not a spatially variable quantity but can be regarded, with respect to the long wave scale, as spatially constant. Its effect on SAR ocean waves imaging, which has been observed in many SAR images, can be described simply as a low-pass azimuthal filter (Seichter 1980, Beal et al. 1983, Monaldo 1984, Hasselmann et al. 1985, Alpers and Brüning 1986). (In the closed nonlinear transfer expression of Hasselmann and Hasselmann (1990), the azimuthal filter term is recovered explicitly as a factor $\exp \left(-k_{\mathrm{x}}^{2}\left\langle\xi^{2}\right\rangle\right)$, where $k_{\mathrm{x}}$ is the azimuthal wave number component and $\left\langle\xi^{2}\right\rangle$ is the total mean square azimuthal displacement due to velocity bunching. Note that $\left\langle\xi^{2}\right\rangle$ is defined here as the integral over the entire wave spectrum, not just over the subresolution scale components. This is in accordance with the empirical findings of Lyzenga (1986) and Beal (personal communication)).

In the Monte-Carlo technique used in this study, we apply the standard SAR imaging model to compute a set of individual SAR images for a series of random realizations of the ocean wave field. Each image is constructed by superimposing the displaced and smeared image computed for each pixel, i.e., each separate SAR resolution cell, of the original scene. The SAR image variance spectrum is then obtained by averaging the individual SAR variance spectra, computed for each individual scene, over a sufficiently large ensemble of wave field realizations (typically 50). The averaged SAR variance spectra then have 100 degrees of freedom.

According to this SAR ocean wave imaging model, the relation between the SAR image intensity $I(\mathbf{x})$ and the surface backscattering cross-section $\sigma(\mathbf{x})$ for a given wave field realization is given by

$$
I(\mathbf{x})=\iint \sigma\left(\mathbf{x}^{\prime}\right)\left(\rho_{\mathrm{a} N}\left(\mathbf{x}^{\prime}\right)\right)^{-1} \exp -\left\{\pi^{2}\left(x-x^{\prime}-\frac{R}{V} \mu_{\mathrm{r}}\left(\mathbf{x}^{\prime}\right)\right)^{2} / \rho_{\mathrm{a} N}^{2}\left(\mathbf{x}^{\prime}\right)\right\} \delta\left(y-y^{\prime}\right) d y^{\prime} d x^{\prime}
$$

where $I(\mathbf{x})$ is defined in the same dimensionless units as $\sigma(\mathbf{x}), \mathbf{x}=(x, y)$ and $\mathrm{x}^{\prime}=\left(x^{\prime}, y^{\prime}\right)$ denote the Cartesian coordinates of the image and the ocean plane, respectively, $R$ is the distance between the antenna and the target and $V$ the platform velocity. The $x$-direction is taken as the azimuthal (satellite flight) direction. The term $(R / V) u_{\mathrm{r}}\left(\mathrm{x}^{\prime}\right)$ represents the Doppler offset arising from the radial orbital (facet) velocities $u_{\mathrm{r}}\left(\mathbf{x}^{\prime}\right)$ of the long ocean waves.

Neglecting the (very small) range-azimuthal coupling, the SAR system is represented as separable in the azimuthal and range channels. With respect to the range resolution, the sea surface can be considered as frozen, since the velocity relevant for range location is the speed of light. To simplify the discussion, the SAR impulse 
response function in the range direction has been simply set equal to the Dirac delta function $\delta\left(y-y^{\prime}\right)$.

The width of the impulse response function in the azimuthal direction is determined by the 'orbital velocity degraded' azimuthal resolution for $N$ incoherent looks $\rho_{\alpha N}\left(\mathbf{x}^{\prime}\right)$ (cf. Raney 1980, Rufenach and Alpers 1981, Alpers and Brüning 1986)

$$
\rho_{\mathrm{a} N}\left(\mathbf{x}^{\prime}\right)=N \rho_{\mathrm{a}}\left[1+\frac{\pi^{2} T^{4}}{N^{2} \lambda_{0}^{2}} a_{\mathrm{r}}^{2}\left(\mathbf{x}^{\prime}\right)+\frac{1}{N^{2}} \frac{(\Delta x)^{2}}{\rho_{\mathrm{a}}^{2}}\right]^{1 / 2}
$$

The second and third terms in the square root expression represent the degradation due to the large-scale orbital acceleration $a_{\mathrm{r}}\left(\mathbf{x}^{\prime}\right)$ and the subresolution scale velocity spread $\Delta x$, respectively. The scale $\rho_{\mathrm{a}}$ denotes the nominal single-look azimuthal resolution

$$
\rho_{\mathrm{a}}=\lambda_{0} R /(2 V T)
$$

where $\lambda_{0}$ is the radar wavelength and $T$ the full-bandwidth, single-look SAR integration time.

For the purposes of this paper we will consider only a simplified multi-look analysis, in which each look is assumed to sample a sea surface with the identical instantaneous long wave field properties, as defined with respect to the centre of look time. Different looks therefore differ only in the different (independent) realizations of the short wave scattering components. Thus we ignore the fact that the long wave components experience a small translation, with their respective phase velocities, from one look to the next. This has led to some confusion in the literature with regard to the significance of the long wave phase velocity in SAR imaging. The phase velocity enters in multi-look image superpositioning but not in the basic single look imaging process. The higher order multi-look aspects have been discussed in detail by Raney and Vachon (1988). We also neglect scanning distortions (cf. Valenzuela 1980, Harger 1980, Plant and Keller 1983). These effects are generally negligible for satellites with small ratios of wave phase velocity to platform velocity but can become important for aircraft SARs. Finally, we do not model clutter noise explicitly in our simulations, since this can be treated theoretically (cf. Alpers and Hasselmann 1982).

Completing our definition of terms in equation (2), $\Delta x$ is proportional to the SAR subresolution scale r.m.s radial orbital velocity $\left\langle\left(u_{\mathrm{r}}\right)^{2}\right\rangle^{1 / 2}$, composed of the contribution to the facet velocities from all waves whose half wavelength is smaller than the SAR resolution cell (see, for example, Tucker 1983, 1985, Hasselmann et al. 1985, Alpers and Brüning 1986),

$$
\Delta x=(2)^{1 / 2} \pi \frac{R}{V}\left\langle\left(u_{\mathrm{r}}\right)^{2}\right\rangle^{1 / 2}
$$

Except for very small wind speeds, the short subresolution scale waves lie in Phillips' equilibrium range of the wave spectrum and one can therefore estimate $\Delta x$ as

$$
\Delta x=\pi \frac{R}{V}\left(\frac{\alpha g}{k_{\mathrm{c}}}\right)^{1 / 2}
$$

where $k_{\mathrm{c}}=\pi /\left(N \rho_{\mathrm{a}} \rho_{\mathrm{r}}\right)^{1 / 2}$ denotes the SAR high wavenumber resolution cut off, $\rho_{\mathrm{r}}$ is the ground range resolution, $g$ the acceleration of gravity and $\alpha$ is Phillips' parameter determining the energy level of the high frequency equilibrium spectrum (Phillips 1977). 


\section{The ocean wave spectrum}

The two-dimensional ocean wave spectrum in this study is modelled by a JONSWAP spectrum $E(\mathbf{k})$ (Hasselmann et al. 1973) with a frequency dependent spreading factor (Mitsuyasu 1975)

$$
E(\mathbf{k})=\frac{\alpha}{2} k^{-4} \exp \left\{-\frac{5}{4}\left(\frac{k}{k_{\mathrm{m}}}\right)^{-2}+\ln \gamma \exp \left[-\frac{\left(k^{1 / 2}-k_{\mathrm{m}}^{1 / 2}\right)^{2}}{2 \sigma_{\mathrm{J}}^{2} k_{\mathrm{m}}}\right]\right\} N(p) \cos ^{2 p}\left(\phi-\phi_{\mathrm{m}}\right)
$$

where $k=|\mathbf{k}|, k_{\mathrm{m}}$ is the peak wavenumber (corresponding to the peak frequency of the JONSWAP frequency spectrum, $\gamma$ the peak enhancement factor, $\phi_{\mathrm{m}}$ the angle between the wave propagation direction and the flight $(x$-)direction, $\sigma$, is a parameter which describes the width of the JONSWAP spectrum,

$$
\sigma_{\mathrm{J}}=\left\{\begin{array}{lll}
\sigma_{\mathrm{a}} & \text { if } \quad k \leqslant k_{\mathrm{m}} \\
\sigma_{\mathrm{b}} & \text { if } \quad k \geqslant k_{\mathrm{m}}
\end{array}\right.
$$

and $N(p)$ is the spreading function normalization factor

$$
N(p)=\frac{1}{\pi^{1 / 2}} \frac{\Gamma(1+p / 2)}{\Gamma(1 / 2+p / 2)}
$$

The exponent $p$ in the spreading function is taken as a function of wavenumber following Mitsuyasu (1975) (see also Hasselmann et al., 1980, Holthuijsen 1981),

$$
p= \begin{cases}0.46\left(k / k_{\mathrm{m}}\right)^{-1.25} p_{\mathrm{m}} & \text { for } k \geqslant k_{\mathrm{m}} \\ 0.46\left(k / k_{\mathrm{m}}\right)^{2.5} p_{\mathrm{m}} & \text { for } k<k_{\mathrm{m}}\end{cases}
$$

where

$$
p_{\mathrm{m}}=11 \cdot 5\left(U / c_{\mathrm{m}}\right)^{-2 \cdot 5}
$$

and $U$ denotes the wind speed at a height of $19.5 \mathrm{~m}$ and $c_{\mathrm{m}}=\left(\mathrm{g} / \mathrm{k}_{\mathrm{m}}\right)^{1 / 2}$ is the phase velocity of the peak wavenumber.

In generating realizations of the surface wave field for the Monte-Carlo simulations, equation (6) is used only up to the SAR separation scale $k_{\mathrm{c}}$. Beyond this cutoff, the velocity bunching mechanism is expressed statistically by the velocity spread term given by equation (5).

The JONSWAP form, equation (6), with appropriate choice of parameters, was used for both windsea and swell simulations. For growing windsea spectra, the JONSWAP parameters $\alpha=0.01, \gamma=3.3$ and $\sigma_{\mathrm{a}}=0.07, \sigma_{\mathrm{b}}=0.09$ were chosen, in accordance with Hasselmann et al. (1973). The same $\sigma_{\mathrm{a}}, \sigma_{\mathrm{b}}$ values were retained also for all other simulations. Fully developed spectra were represented by the PiersonMoskowitz spectrum, with $\alpha=0.0081$ and $\gamma=1$. Swell was described by a highly peaked JONSWAP spectrum with $\gamma=10$ and variable energy levels given by $\alpha=4,2,1$ and $0.25 \times 10^{-3}$. The scaling parameter $k_{\mathrm{m}}$ was varied in all simulations.

The dependence on the directional distribution was not systematically explored, as it was found that the simulations were generally rather insensitive to the precise form assumed for the spreading function. A marked spreading function dependence was found only for very narrow band swell (where a narrower distribution than the form of equation (9) is generally appropriate). Systematic simulation runs covering the imaging parameter phase space were carried out for a fully developed Pierson-Moskowitz spectrum. The other spectral forms were used only in exploratory simulations to test the sensitivity of the results on the assumed spectral shape. 


\section{Non-dimensional imaging parameters}

To systematize the results of the simulation experiments, it is useful to introduce non-dimensional parameters characterizing the various SAR imaging processes.

\subsection{Velocity bunching parameter}

The modulation of the SAR image intensity induced by velocity bunching is proportional to the gradient of the radial orbital velocity in the azimuthal direction, $(\partial / \partial x) \mathrm{u}_{\mathrm{r}}(\mathbf{x})$. The process can be described by a linear modulation transfer function (MTF) if $\left|(R / V)(\partial / \partial x) u_{\mathrm{r}}(\mathbf{x})\right|<<1$ (Alpers et al. 1981), and is strongly nonlinear if the reverse inequality holds.

A useful parameter describing the degree of nonlinearity is the non-dimensional velocity bunching parameter (Alpers 1983):

$$
C=\frac{R}{V}\left\langle\left(\frac{\partial}{\partial x} u_{\mathrm{r}}(\mathbf{x})\right)^{2}\right\rangle^{1 / 2} \approx C^{\max } \cos \Phi_{\mathrm{m}}
$$

where

$$
C^{\max }=\frac{R}{4 V} g^{1 / 2} k_{\mathrm{m}}^{3 / 2} H_{s} \cos \theta
$$

Here $H_{\mathrm{s}}$ denotes the significant waveheight, $\Phi_{\mathrm{m}}$ is the propagation direction of the peak wave component $k_{\mathrm{m}}$ relative to the azimuth direction and $\theta$ is the incidence angle. The parameter $C^{\max }$ represents the ratio of the azimuthal displacement induced by the orbital velocity to the dominant wave length of the spectrum. $C$ represents the corresponding ratio with respect to the longer effective wavelength $\lambda_{x}=2 \pi / k_{x}$ for a wave profile along the azimuthal direction. For waves propagating in range direction, $C$ becomes zero, i.e. the orbital motion effects vanish and the waves are imaged linearly. The maximum nonlinearity occurs for waves propagating in azimuthal direction, $C=C^{\max }$. For fully developed windseas, equation (12) implies that $C$ is proportional to $k_{\mathrm{m}}^{1 / 2}$ or $U^{-1}$, since in this case $H_{\mathrm{s}} \sim k_{\mathrm{m}}$ and $k_{\mathrm{m}} \sim U^{-2}$. Thus for fully developed windseas, the nonlinearity decreases with increasing wind speed. Although contrary to immediate intuition, this can be readily understood as a result of the increasing wavelength of the windsea, which increases more rapidly with wind speed $\left(\sim U^{2}\right)$ than the r.m.s. orbital velocity $(\sim U)$.

\subsection{Velocity spread parameter}

A non-dimensional velocity spreading parameter $A_{v}$ characterizing the degradation of the azimuthal resolution due to variations of the sub-resolution scale facet velocities (cf. equations (2) and (4)) can be defined as

$$
A_{v}=\Delta x / \rho_{\mathrm{a}}=2^{1 / 2} \pi \frac{R}{V}\left\langle\left(u_{\mathrm{r}}\right)^{2}\right\rangle^{1 / 2} / \rho_{\mathrm{a}} \approx \frac{2 \pi T}{\lambda_{0}}\left\{\frac{a g}{k_{\mathrm{c}}}\right\}^{1 / 2}
$$

For a short wave spectrum, which could be severely degraded by the azimuthal cutoff, a more relevant parameter than the ratio $\Delta x / \rho_{\mathrm{a}}$ is the ratio

$$
F_{v}=\Delta x / \lambda_{\mathrm{m}}
$$

of the azimuthal image smearing scale to the ocean peak wavelength $\lambda_{\mathrm{m}}$. Values of $F_{v}$ of order unity imply that the velocity spread significantly attenuates the image spectrum not only at high wave numbers but also in the region of the spectral peak.

In addition to the velocity spread term, the image is smeared by the second-order 
orbital acceleration. However, although this term is included in the computations, it was found to be small compared with the velocity spread contribution. Thus we have not stratified the date with respect to this effect and need not introduce a corresponding non-dimensional acceleration parameter.

\subsection{Signal-to-clutter-noise ratio}

As already mentioned, clutter noise is not explicitly included in the present SAR imaging model (equation (1)), as this can be readily computed theoretically (Alpers and Hasselmann 1982). In the low wavenumber domain the clutter spectrum is white and is given by

$$
G_{\mathrm{cl}}^{0}=\left(\frac{2}{\pi}\right)^{1 / 2} \rho_{\mathrm{a}}
$$

The signal-to-clutter ratio $\mathrm{S} / \mathrm{C}$ is defined here in terms of a peak to background ratio as

$$
\mathrm{S} / \mathrm{C}=10 \log \left(F^{\max } / G_{\mathrm{cl}}^{0}\right)
$$

where $F^{\max }$ is the SAR energy density of the spectral peak. In the linear regime S/C can be shown to be approximately proportional to the parameter

$$
B=\left(k_{\mathrm{m}} \rho_{\mathrm{s}}\right)^{-1}
$$

which we shall therefore use as an alternative simple non-dimensional parameter characterizing the signal-to-clutter ratio.

Another parameter which is useful for characterizing the signal-to-clutter ratio is the shortest wavelength $\lambda_{\mathrm{cl}}$ which can still be detected before the SAR spectral signal merges into the clutter background spectrum, or the dimensionless ratio

$$
\lambda_{\mathrm{cl}}^{d}=\lambda_{\mathrm{cl}} / 2 N \rho_{\mathrm{a}}
$$

where $N \rho_{\mathrm{a}}$ is the theoretical SAR resolution (without motion degradation) for $N$ looks.

\subsection{Distortion parameters}

To characterize the nonlinear image distortions we introduce the following nondimensional parameters:

(1) the stretching parameter for the peak wavelength,

$$
S=\lambda_{\mathrm{m}}^{\mathrm{s}} / \lambda_{\mathrm{m}}^{o}
$$

where $\lambda_{\mathrm{m}}^{\mathrm{s}}, \lambda_{\mathrm{m}}^{0}$ denote the wavelengths of the spectral peaks in the SAR image and ocean planes, respectively:

(2) the rotation parameter for the propagating direction of the peak wavenumbers,

$$
\Delta \Phi=\Phi_{\mathrm{m}}^{\mathrm{s}}-\Phi_{\mathrm{m}}^{0}
$$

where $\Phi_{\mathrm{m}}^{\mathrm{s}}, \Phi_{\mathrm{m}}^{0}$ denote the azimuthal angles of the spectral peaks in the SAR image and ocean planes, respectively; and

(3) the signal-to-clutter ratio ( $\mathrm{S} / \mathrm{C})$, equation (16) and the dimensionless azimuthal signal-to-clutter cut-off wavelength $\lambda_{\mathrm{cl}}^{\mathrm{d}}$, equation (18). 


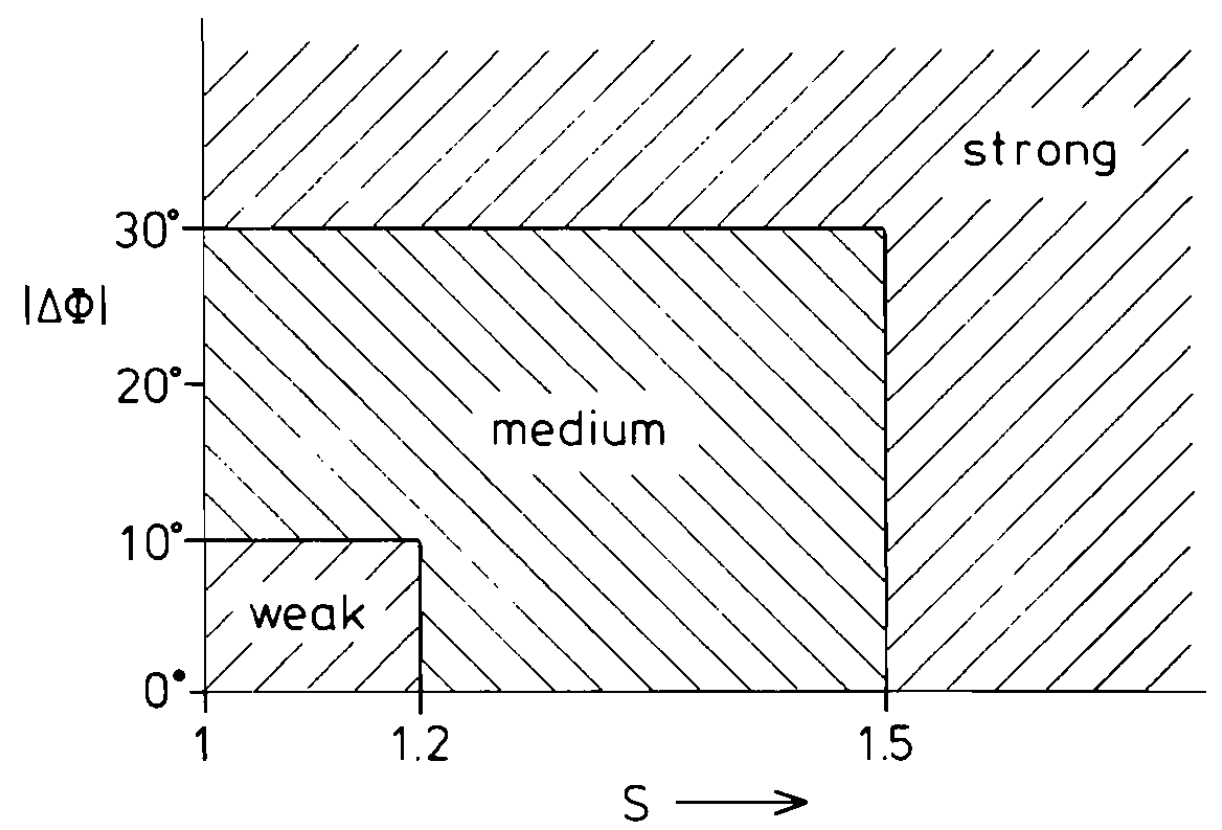

Figure 1. Region of nonlinearity in the distortion parameter plane $S$ (peak wave length stretching) and $|\Delta \Phi|$ (rotation of peak wavenumber direction).

The stretching and the rotation parameters, $S$ and $\Delta \Phi$, can be used to define the regimes where the SAR imaging process is weakly, medium or strongly nonlinear (see figure 1).

\section{Linear SAR imaging}

\subsection{RAR modulation transfer function}

To a good approximation, it can be assumed that for a 'frozen sea surface' the real aperture radar (RAR) cross-section fractional modulation $m(\mathbf{x})=\sigma(\mathbf{x}) / \bar{\sigma}(\bar{\sigma}=$ mean of $\sigma)$ is linearly related to the long ocean wave field. In this case the imaging can be described in the Fourier domain by a complex dimensionless modulation transfer function $M^{\mathrm{RAR}}(\mathbf{k})$ (Alpers et al. 1981, Feindt et al. 1986)

$$
m(\mathbf{k})=\sigma(\mathbf{k}) / \bar{\sigma}=k M^{\mathrm{RAR}}(\mathbf{k}) Z(\mathbf{k})+k M^{\mathrm{RAR}}(-\mathbf{k}) Z(-\mathbf{k})
$$

Here $m(\mathbf{k}), \sigma(\mathbf{k})$ denote the Fourier transforms of $m(\mathbf{x}), \sigma(\mathbf{x})$, respectively and $Z(\mathbf{k})$ is the Fourier wave component of the ocean surface elevation $\xi(\mathbf{x}, t)$.

$$
\begin{aligned}
\sigma(\mathbf{x}) & =\bar{\sigma}+\int \sigma(\mathbf{k}) \exp (i \mathbf{k x}) d \mathbf{k} \\
m(\mathbf{x}) & =\int m(\mathbf{k}) \exp (i \mathbf{k x}) d \mathbf{k} \\
\xi(\mathbf{x}, t) & =\int(Z(\mathbf{k}) \exp i(\mathbf{k} \mathbf{x}-\omega t)+\text { compl. conj. }) d \mathbf{k}
\end{aligned}
$$

where $\omega=\sqrt{ } g k$. 
Note that in contrast to equations (22), $Z(\mathbf{k})$ contains information on the direction of wave propagation. The Fourier amplitudes $Z(\mathbf{k})$ must be distinguished from the Fourier amplitudes of the transform of the frozen surface at time $t=0$, which would be given by $Z(\mathbf{k})+Z(-\mathbf{k})^{*}$. We shall in fact consider the surface only at time $t=0$, but still need the full time representation in order to consider both the instantaneous surface elevation and surface orbital velocity.

The (two sided) variance spectrum of the cross-section modulation $F(\mathbf{k})$ and surface wave spectrum $E(\mathbf{k})$ are related to the (statistically independent) Fourier amplitudes through

$$
\left\langle m\left(\mathbf{k}_{1}\right) m\left(\mathbf{k}_{2}\right)^{*}\right\rangle=F(\mathbf{k}) \delta\left(\mathbf{k}_{1}-\mathbf{k}_{2}\right)
$$

and

$$
2\left\langle Z\left(\mathbf{k}_{1}\right) Z\left(\mathbf{k}_{2}\right)^{*}\right\rangle=E(\mathbf{k}) \delta\left(\mathbf{k}_{1}-\mathbf{k}_{2}\right)
$$

The relation between the RAR image spectrum and surface wave spectrum is thus given by

$$
F(\mathbf{k})=\frac{k^{2}}{2}\left(\left|M^{\mathrm{RAR}}(\mathbf{k})\right|^{2} E(\mathbf{k})+\left|M^{\mathrm{RAR}}(-\mathbf{k})\right|^{2} E(-\mathbf{k})\right)
$$

The RAR modulation transfer function $M^{\text {RAR }}$ consists of the sum of the complex nondimensional hydrodynamic and tilt MTFs,

$$
M^{\mathrm{RAR}}=M^{\mathrm{hydr}}+M^{\mathrm{tilt}}
$$

The tilt contribution to $M^{\text {RAR }}$ can be computed theoretically but the hydrodynamic MTF is only poorly known. In the present study we assume that $M^{\text {RAR }}$ is independent of $k$ and that the azimuthal dependence of the modulus and phase of $M^{\mathrm{RAR}}$ is given by

$$
\left|M^{\mathrm{RAR}}\left(\Phi_{\mathrm{m}}\right)\right|=0 \cdot 5\left|M_{\mathrm{r}}^{\mathrm{RAR}}\right|\left[1+\sin ^{2} \Phi_{\mathrm{m}}\right]
$$

and

$$
\eta^{\mathrm{RAR}}=\eta^{\mathrm{RAR}} \sin ^{2} \Phi_{\mathrm{m}}
$$

where $M_{\mathrm{r}}^{\mathrm{RAR}}$ and $\eta_{\mathrm{r}}^{\mathrm{RAR}}$ are constants. We have set $M_{\mathrm{r}}^{\mathrm{RAR}}=5$ and $\eta_{\mathrm{r}}^{\mathrm{RAR}}=45^{\circ}$ for most simulations.

The expressions (28), (29), with these values of the constants, are fairly representative of the results obtained in backscattering experiments in the open ocean and windwave tank measurements (cf. Feindt 1985, Wright et al. 1980, Schroeter et al. 1986). Equation (28) implies a ratio of $2: 1$ for the upwind/downwind to the crosswindcross-sections. A phase dependence of the form (29) results if the maximum hydrodynamic modulation occurs near the crests of the long ocean waves, so that the phase $\eta^{\text {hydr }}$ of the hydrodynamic MTF is approximately zero (Wright et al. 1980, Plant and Keller 1983). In this case $\eta^{\text {RAR }}$ is determined largely by $\eta^{\text {tilt }}$, which is in phase with the long wave slope. Note that the sign of $\eta^{\text {RAR }}$ depends on the ocean wave propagation direction relative to the radar look direction and is negative if the waves propagate towards the radar. In general it was found that the imaging is not very sensitive to the form assumed for $M^{\text {RAR }}$ except for some interference effects with the linear velocity bunching MTF in the linear regime (cf. following sub-section and $\S 7$ ).

\subsection{Velocity bunching transfer function}

In the linear imaging regime, the velocity bunching mechanism can be described by a linear modulation transfer function, $M^{\mathrm{vb}}$, in analogy with the RAR modulation 
transfer function (Alpers et al., 1981, and many others),

$$
M^{\mathrm{vb}}(\mathbf{k})=\frac{R}{V} \frac{k_{x}}{k} \omega\left(\cos \theta-i \frac{k_{y}}{k} \sin \theta\right)
$$

The first term in equation (30) arises from the vertical velocity component, while the second term, in quadrature with the first, from the horizontal velocity component. For steep incidence angles $\theta$, appropriate for satellite SAR imaging, the maximum of $M^{\mathrm{vb}}$ is encountered for waves propagating in the azimuthal direction, $\Phi_{\mathrm{m}}=0^{\circ}$ :

$$
\left|M^{\mathrm{vb}}\right|^{\max } \approx \frac{R}{V} g^{1 / 2} k^{1 / 2} \cos \theta
$$

\subsection{SAR modulation transfer function}

In the linear imaging regime, the net SAR modulation transfer function, $M^{\text {SAR }}$, is given by the complex sum

$$
M^{\mathrm{SAR}}=M^{\mathrm{RAR}}+M^{\mathrm{vb}}
$$

The square modulus is accordingly

$$
\left|M^{\mathrm{SAR}}\right|^{2}=\left.M^{\mathrm{RAR}}\right|^{2}+\left|M^{\mathrm{vb}}\right|^{2}+2\left|M^{\mathrm{RAR}}\right|\left|M^{\mathrm{vb}}\right| \cos \left(\eta^{\mathrm{vb}}-\eta^{\mathrm{RAR}}\right)
$$

$\left|M^{\text {SAR }}\right|$ depends strongly on the azimuth angle $\Phi_{\mathrm{m}}$, as seen in the examples shown in figure 2 for the cases $\left|M^{\mathrm{vb}}\right|^{\max }=0.5,7,0.6,15 \cdot 3$ (see equation (31)). The SAR MTF exhibit minima near the range direction $\left(\Phi_{\mathrm{m}}=90^{\circ}, 270^{\circ}\right)$ since the velocity bunching contribution (equation (30)) vanishes for these directions. Although the (moduli of the) RAR and velocity bunching transfer functions are separately symmetric about the range axis, the net SAR MTF is in general not symmetric about this axis because of interference between the complex velocity bunching and RAR modulation transfer functions (figure 3). For the RAR phase relation (equations (28) and (29)) chosen for most of our computations, the modulations add constructively in the range $-90^{\circ}<\eta<90^{\circ}$, where $\eta=\eta^{\mathrm{vb}}-\eta^{\mathrm{RAR}}$, and destructively otherwise.

\section{Monte-Carlo simulations}

To compute the transformation from the surface wave spectrum to the SAR image spectrum, individual SAR images were computed for an ensemble of realizations of the surface wave field. Each SAR image was Fourier analysed, and the statistical image variance spectrum was then estimated by averaging the square modulus of the Fourier amplitudes of the individual image spectra over the ensemble of realizations.

The individual surface wave realizations were simulated by superimposition of a discrete two-dimensional amplitude spectrum of sine and cosine surface wave components, each Fourier component being generated by a Gaussian random number generator whose variance was normalized by the spectral energy contained in that discrete spectral bin.

The SAR image for an individual surface wave realization was computed using the basic mapping relation (1). The spatial cross-section field was obtained by inversion of the Fourier transform, which was determined using equations (21), (22), (28) and (29). The range velocity and range acceleration fields were similarly constructed from the Fourier representations, using the transfer function relations of standard linear wave theory,

$$
\begin{aligned}
& v_{\mathrm{r}}(\mathbf{k})=-i \omega D(\mathbf{k}) Z(\mathbf{k}) \\
& a_{\mathrm{r}}(\mathbf{k})=-\omega^{2} D(\mathbf{k}) Z(\mathbf{k})
\end{aligned}
$$




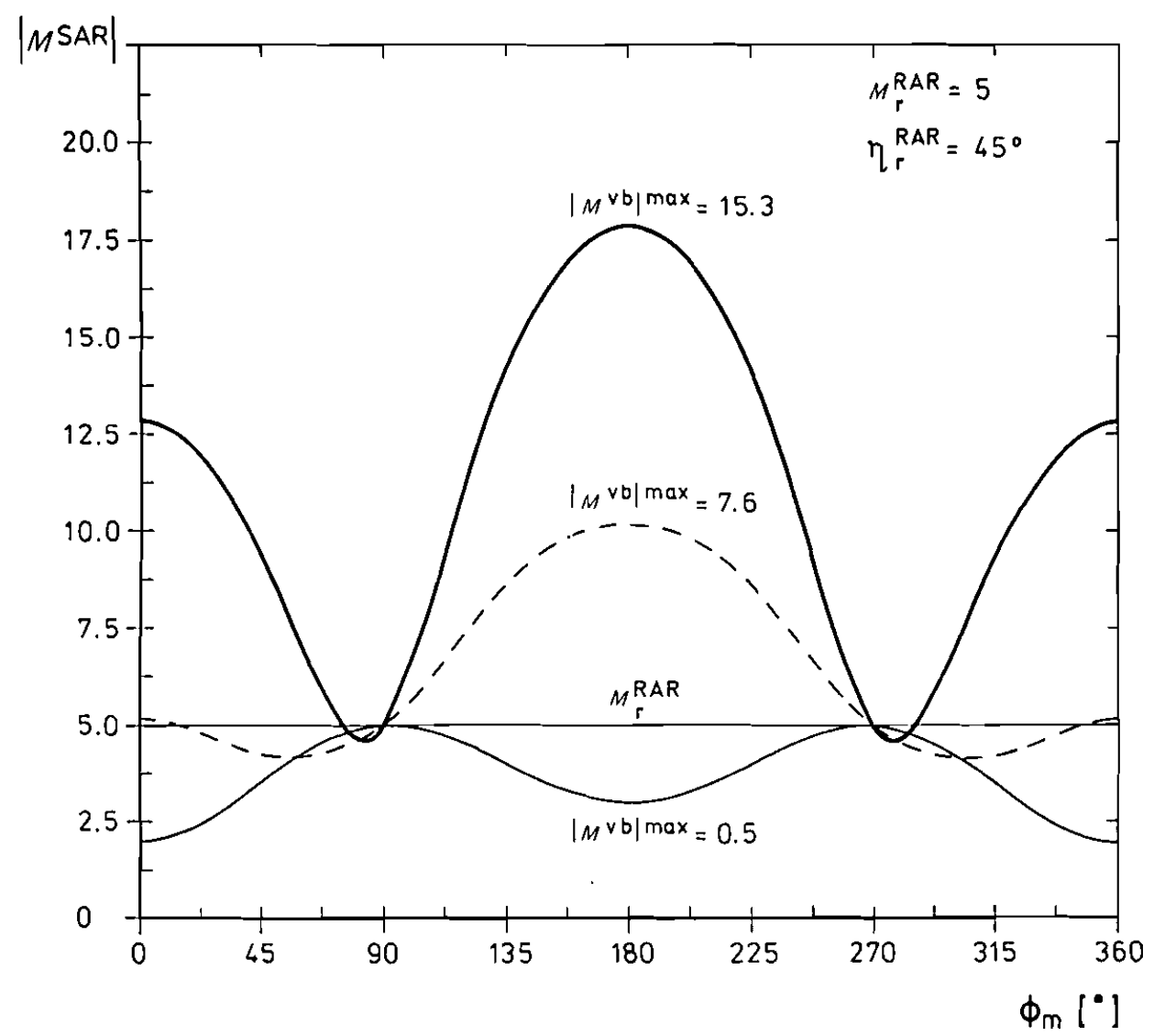

Figure 2. The modulus of the dimensionless SAR modulation transfer function $\left|M^{\text {SAR }}\right|$ as a function of azimuth angle $\Phi_{\mathrm{m}}^{\circ}$ for a fixed RAR MTF (linear imaging).

where the factor

$$
D(\mathbf{k})=\cos \theta-i\left(k_{y} / k\right) \sin \theta
$$

represents the ratio of the range component of the orbital velocity and acceleration vectors relative to the vertical components.

For $\lambda_{\mathrm{m}}<200 \mathrm{~m}$, a two-dimensional array of $128 \times 128$ pixels with a pixel size of $12 \mathrm{~m} \times 12 \mathrm{~m}$, was used, yielding a $1536 \mathrm{~m} \times 1536 \mathrm{~m}$ scene. For $\lambda_{\mathrm{m}} \geqslant 200 \mathrm{~m}$ the pixel size was doubled to $24 \mathrm{~m} \times 24 \mathrm{~m}(3072 \mathrm{~m} \times 3072 \mathrm{~m}$ scene $)$. The SAR image variance spectrum was smoothed by applying a 3 pixel Hanning filter in the range wavenumber direction.

The SAR and ocean wave parameters used for most of the simulations are listed in tables $l$ and 2. The largest $R / V$ value of $128 \mathrm{~s}$ applies for Seasat, while $R / V=0$ corresponds to a RAR. The ocean wave parameters refer to a fully developed Pierson-Moskowitz windsea, while the ocean peak wavelength $\lambda_{m}$ was varied through the values $100 \mathrm{~m}, 200 \mathrm{~m}$ and $400 \mathrm{~m}$. The azimuth angle $\Phi_{\mathrm{m}}$ was varied in the range $0^{\circ}-180^{\circ}$ in steps of $30^{\circ}$ (since the SAR modulation transfer function $M^{\mathrm{SAR}}$ is symmetric about $\Phi_{\mathrm{m}}=0$, only the half space $0^{\circ}<\Phi_{\mathrm{m}}<180^{\circ}$ need be considered, cf. figure 2). 


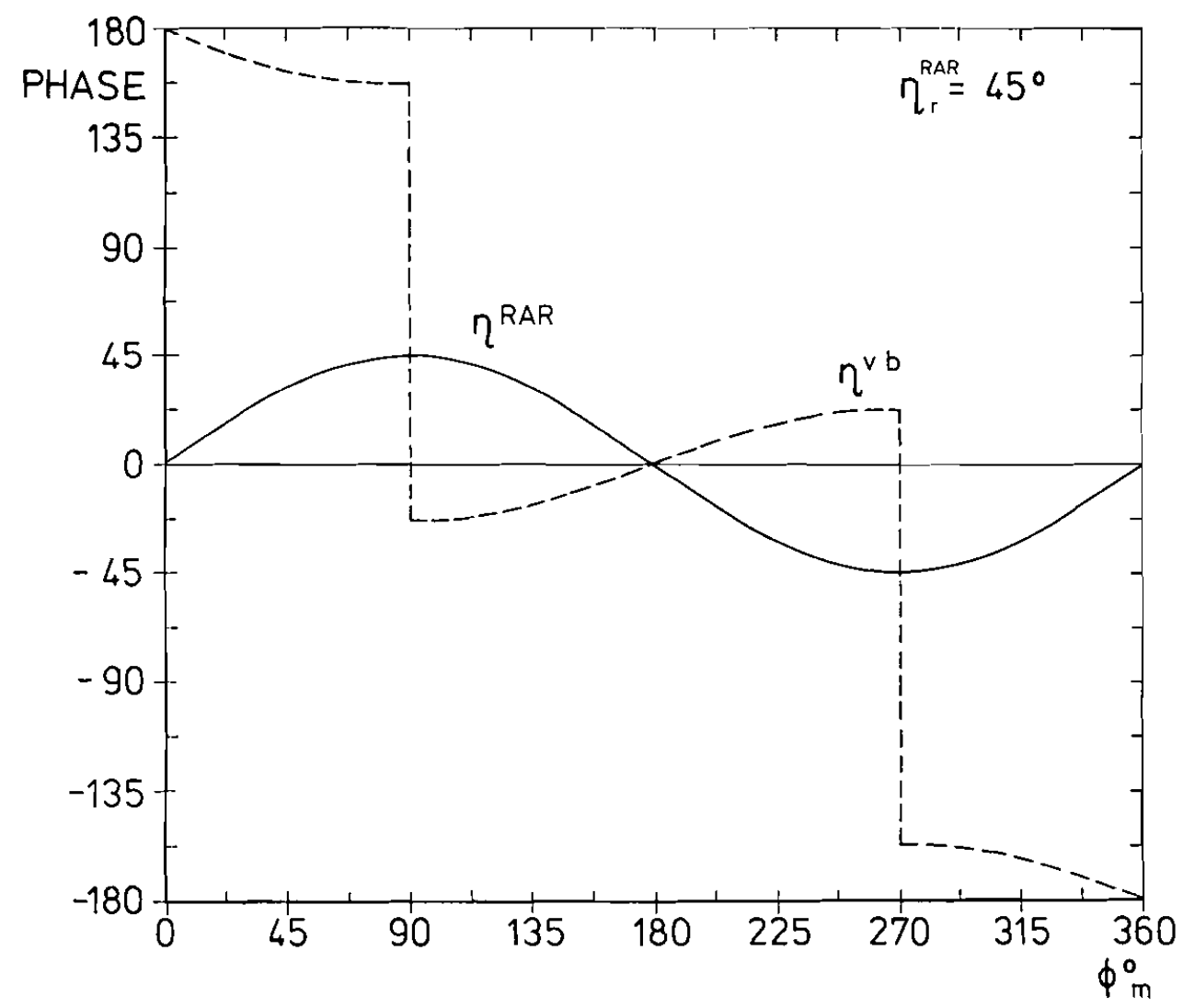

Figure 3. Phases $\eta^{\mathrm{RAR}}$ of the RAR MTF and $\eta^{\mathrm{vb}}$ of the velocity bunching MTF as a function of the azimuth angle $\Phi_{\mathrm{m}}$.

Table 1. SAR parameters used in the core simulations

\begin{tabular}{ccccccc}
\hline $\begin{array}{c}\lambda_{0} \\
(\mathrm{~m})\end{array}$ & $\begin{array}{c}S_{\mathrm{a}} \\
(\mathrm{m})\end{array}$ & $N$ & $\begin{array}{c}R / V \\
(\mathrm{~s})\end{array}$ & $\begin{array}{c}0 \\
\left({ }^{\circ}\right)\end{array}$ & $\cdot M_{\mathrm{r}}^{\text {RAR }}$ & $\begin{array}{c}\eta^{\text {RAR }} \\
\left({ }^{\circ}\right)\end{array}$ \\
\hline 0.235 & 6.25 & 4 & 0 & 23 & 5 & 45 \\
& & & 15 & & & \\
& & 60 & & & \\
& & 128 & & & \\
\hline
\end{tabular}

Table 2. Ocean wave parameters used in the core SAR simulation

\begin{tabular}{cccc}
\hline$\alpha$ & $\gamma$ & $\lambda_{\mathrm{m}}(\mathrm{m})$ & $\Phi_{\mathrm{m}}\left({ }^{\circ}\right)$ \\
\hline 0.0081 & 1.0 & 100 & 0 \\
& & & 30 \\
& & & 60 \\
& & 90 \\
& & 120 \\
& & 150 \\
& & 180 \\
\hline
\end{tabular}


In addition to the 'core' simulation cases listed in tables 1 and 2, simulation runs were also carried out for sea states corresponding to typical growing. windseas $\left(\gamma=3.3, \alpha=0.01, \lambda_{\mathrm{m}}=200 \mathrm{~m}\right.$, with $\left.R / V=128 \mathrm{~s}\right)$ and narrow peak ocean swells, to assess the dependence of the results on the form assumed for the wave spectrum (cf. $\S 3)$. Similarly, to investigate the sensitivity of the SAR imaging process on the form assumed for the RAR MTF, a series of simulations were also carried out with $\left|M^{\mathrm{RAR}}\right|=5,10,15$ and $\eta^{\mathrm{RAR}}=0^{\circ}, 45^{\circ}, 90^{\circ}$ for fixed values of $C^{\max }\left(C^{\max }=2 \cdot 3,1 \cdot 1,0 \cdot 55\right)$ and $\Phi_{\mathrm{m}}\left(\Phi_{\mathrm{m}}=0^{\circ}, 30^{\circ}, 60^{\circ}, 90^{\circ}, 120^{\circ}, 150^{\circ}\right)$. The variance spectra were computed by averaging over 50 Monte-Carlo simulations (100 degrees of freedom).

Examples of two-dimensional Monte-Carlo simulations are presented in figures 4-6 for the case of a fully developed windsea with peak wavelength $\lambda_{\mathrm{m}}=2 \pi / k_{\mathrm{m}}=100 \mathrm{~m}$ and varying $R / V$ values and propagation directions. For $\lambda_{m}=100 \mathrm{~m}$ the $R / V$ values listed above correspond to the nonlinear (velocity bunching) parameter values $C^{\max }=3 \cdot 1,1 \cdot 5,0 \cdot 7,0 \cdot 4,0((b)-(f)$ respectively; $(a)$ represents the ocean wave spectrum itself). A comparison of the simulations for the three different propagation directions shown in figures $4-6\left(\Phi_{\mathrm{m}}=0^{\circ}\right.$ : azimuthally propagating waves; $\Phi_{\mathrm{m}}=60^{\circ}$ and $\Phi_{\mathrm{m}}=90^{\circ}$ : range propagating waves) illustrates the strong influence of the wave propagation direction on the image spectrum.

A general feature of all SAR image spectra in the nonlinear regime is the strong distortion into cigar shaped distributions (Seichter 1980). The spectral energy is shifted towards lower azimuthal wavenumbers, resulting in a rotation of the SAR image spectrum towards the range direction. In addition, the SAR spectra show a sharp cut-off in azimuth and a broadening in the range direction. As $C^{\max }$ and $A_{v}$ are gradually decreased $((c)-(f)$ of figures 4-6) the SAR image spectra becomes less distorted. The imaging process is essentially linear for $C^{\max }<1$ for range-travelling waves. In this regime, an asymmetry of the SAR image spectrum about the range direction may occur due to the interference of the complex RAR and velocity bunching MTFs.

For spectra with a broad peak in the range direction, a single peak windwave spectrum can be mapped into a double-peaked SAR image spectrum (cf. figures 5 and 6). This is because the velocity bunching mechanism, which dominates the imaging in most cases, vanishes for the range direction but rapidly takes on large values on either side of this direction (cf. figure 2). Thus a rather sharp valley is normally cut into the SAR spectrum along the range axis. The effect has been observed in SAR variance spectra obtained from the Shuttle Imaging Radar-B (SIR-B) experiment (Brüning et al. 1988).

In summary, figures 4-6 indicate a strong dependence of the SAR imaging mechanism both on the nonlinearity parameter $C^{\max }$ and on the direction of ocean wave propagation $\Phi_{m}$ relative to the SAR flight direction. This is to be expected, as the dominant motion effects, velocity bunching and velocity spread, are proportional to $C^{\max }$, and all contributions to the imaging process (velocity bunching, hydrodynamic and tilt modulation and image smear due to orbital acceleration and subresolution scale velocity spread) depend strongly on the azimuth angle.

\section{Summary of Monte-Carlo simulations}

In the following sections we summarize the results of the SAR simulation studies for fully developed windseas in terms of the non-dimensional parameters defined in equations (12), (13), and (14). The results are approximately valid also for growing windseas and swells, for the same non-dimensional scaling parameters, provided the 

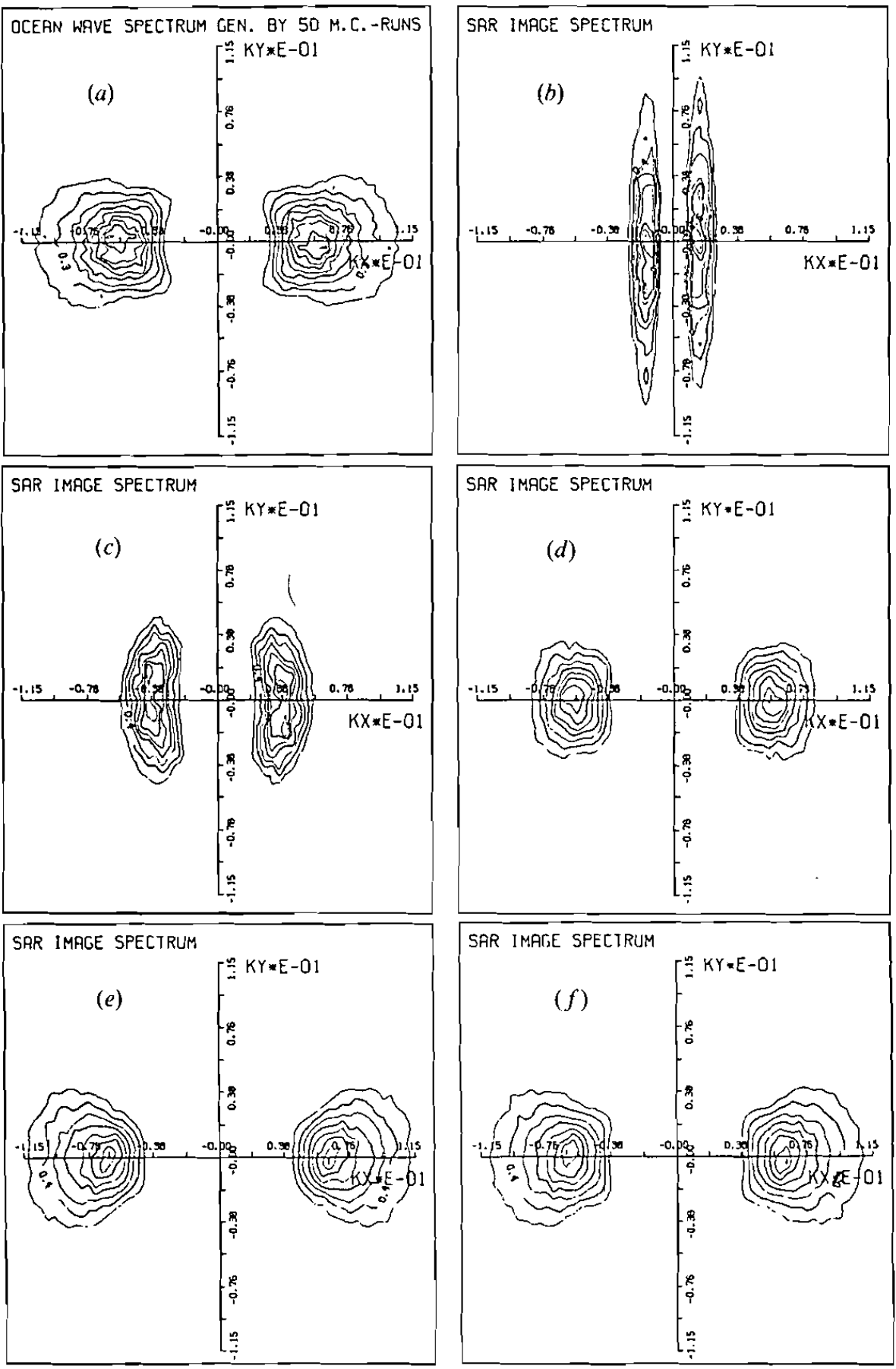

Figure 4. Examples of SAR imaging of a fully developed windsea with a peak wavelength of $100 \mathrm{~m}$ propagating in flight direction (zero azimuth angle) for different $R / V$ ratios. (a), the ocean wave spectrum generated by 50 Monte-Carlo simulation runs. $(b)-(f)$, the SAR image spectra for the nonlinearity (velocity bunching) parameter values $C^{\max }=3 \cdot 1$, $1 \cdot 5,0 \cdot 7,0 \cdot 4,0 \cdot 0$, respectively (the corresponding values of $A_{v}$ are $51,24,12,6,0$ ). 

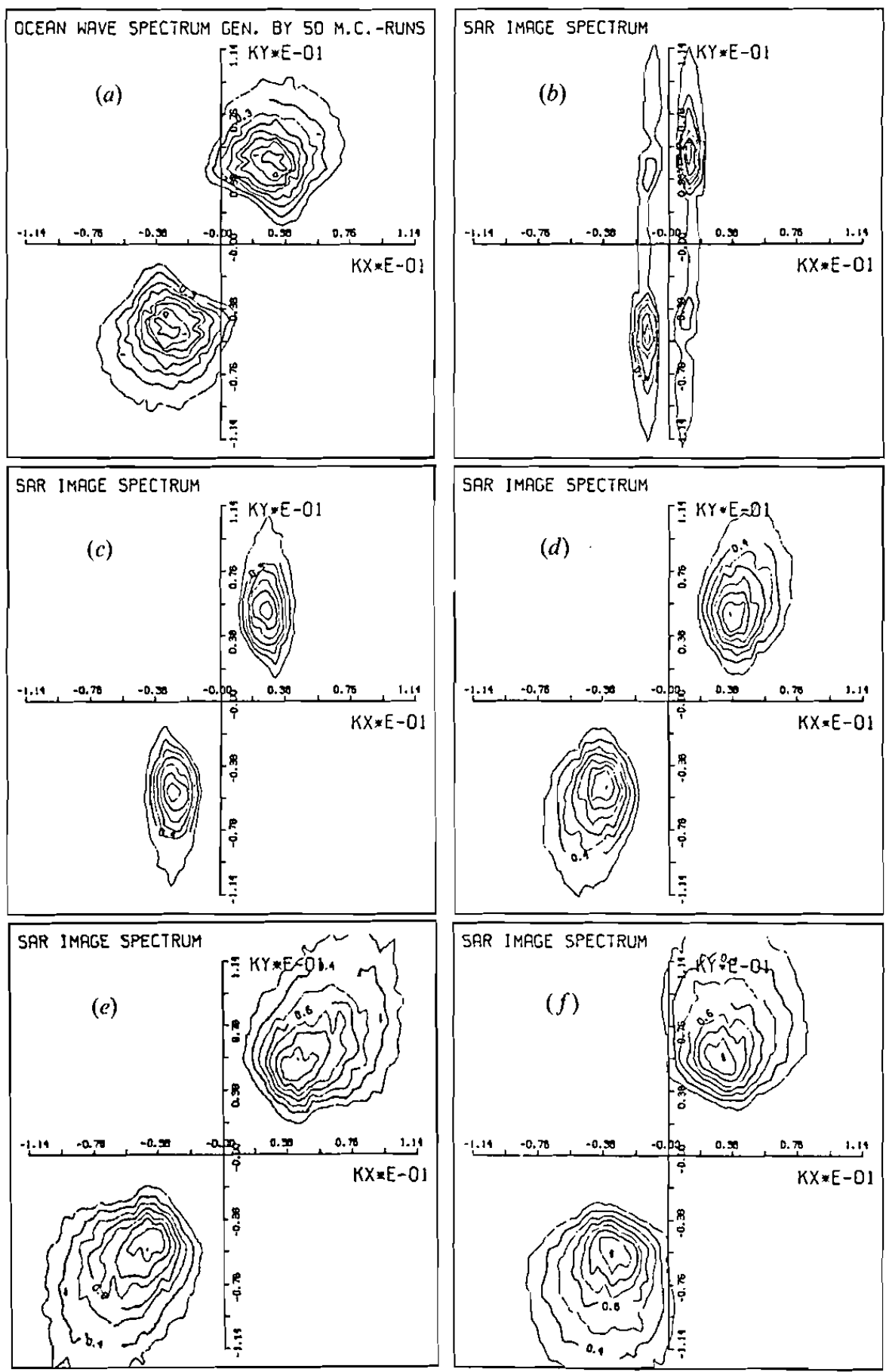

Figure 5. Same as figure 4, but for an azimuth angle of $60^{\circ}$. 

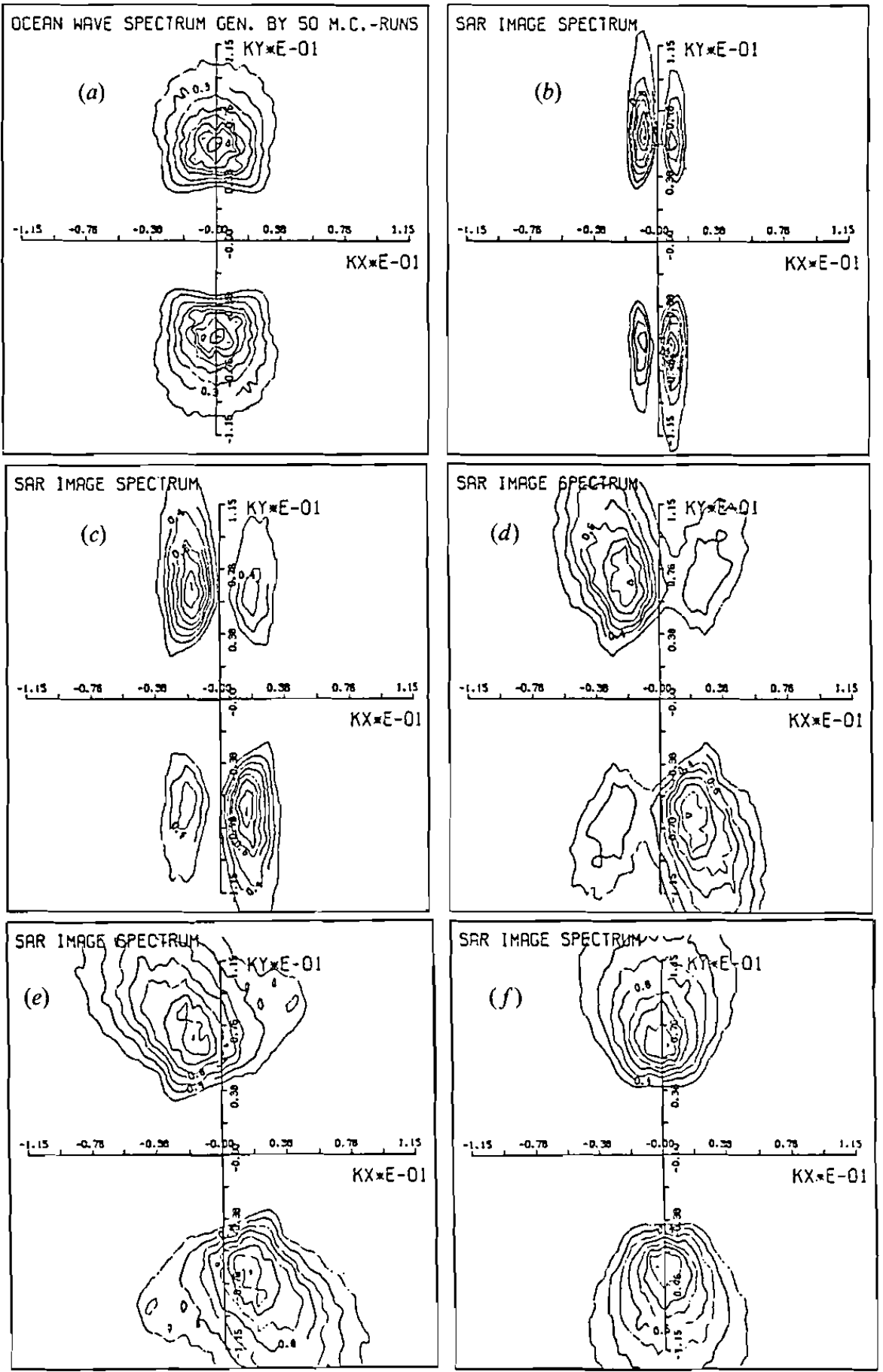

Figure 6. Same as figure 4, but for an azimuth angle of $90^{\circ}$ (range travelling waves). 
spectral shape does not differ too strongly from the Pierson-Moskowitz fully developed windsea spectrum.

The data shown in figure 6-17 are based on a total of 105 numerical experiments, each consisting of 50 Monte-Carlo simulations. To characterize the statistical estimation uncertainties arising from the limitation to a finite number of Monte-Carlo simulations for each parameter setting, r.m.s. errors were computed from a sample of ten. independent numerical simulations, each consisting of 50 Monte-Carlo realizations.

\subsection{Stretching parameter}

The stretching parameter $S$ (equation (19)) is a function of the velocity bunching parameter $C^{\max }$ (equation (12)), the dominant ocean wave propagation direction $\Phi_{\mathrm{m}}$, and the azimuthal smearing parameter $A_{v}$ (equation (13)). Figure 7 shows the

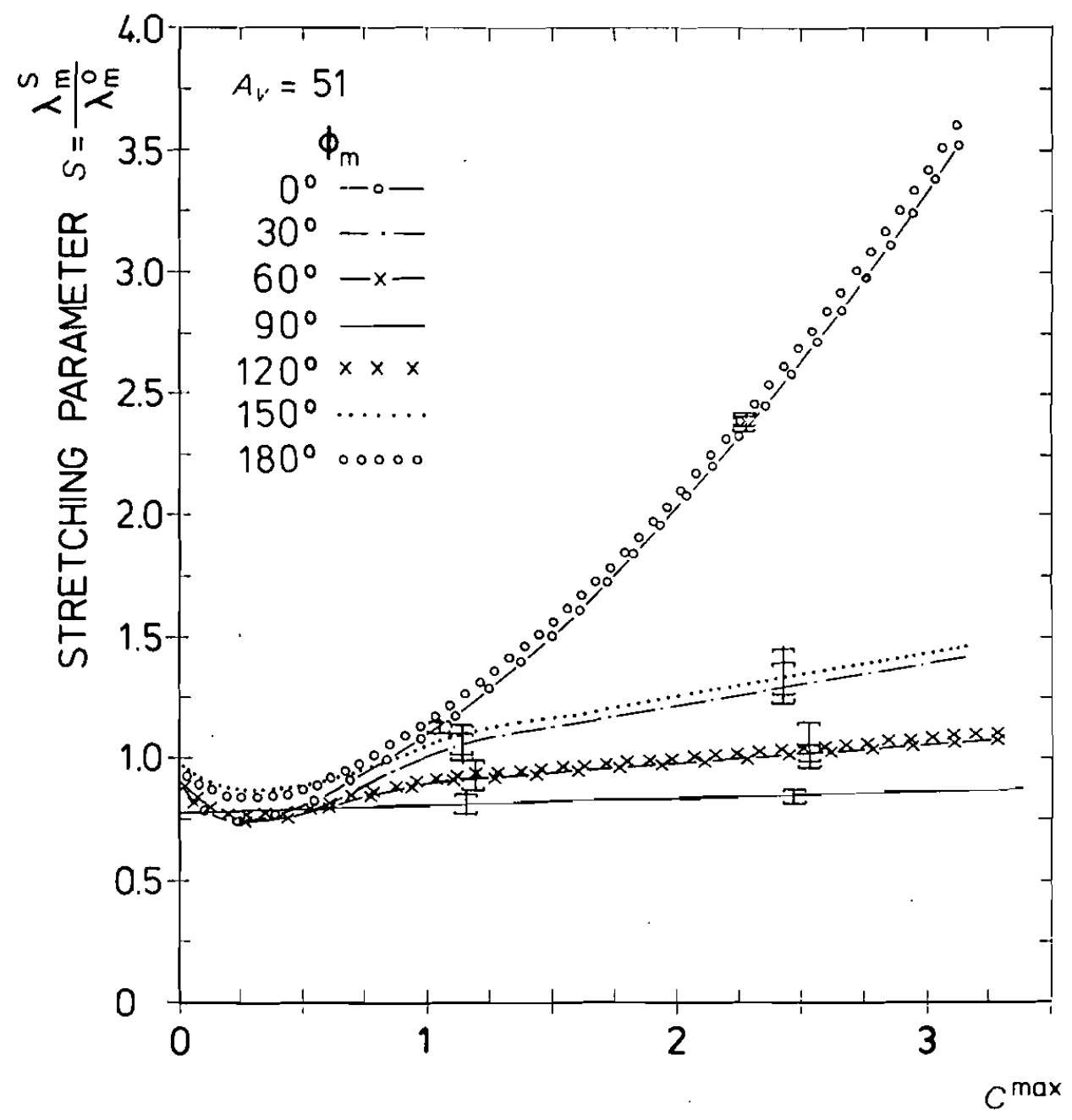

Figure 7. Stretching parameter $S=\lambda_{\mathrm{m}}^{\mathrm{s}} / \lambda_{\mathrm{m}}^{0}$ as a function of the nonlinearity (velocity bunching) parameter $C^{\max }$ for different azimuth angles $\Phi_{\mathrm{m}}$ and fixed velocity spread parameter $A_{\mathrm{v}}=51$. 
dependence of $S$ on $C^{\max }$ and $\Phi_{\mathrm{m}}$ for fixed $A_{v}=51$ (varying $C^{\max }$ for fixed $A_{v}$ corresponds to changing the peak wavelength for fixed $R / V$ ). A significant stretching of the peak wavelength occurs for high values of $C^{\max }$ but only in a small direction segment around the zero azimuth direction $\left(-20^{\circ}<\Phi_{\mathrm{m}}<20^{\circ}\right)$. In the range $30^{\circ}<\Phi_{\mathrm{m}}^{0}$ $<150^{\circ}$ the stretching parameter $S$ remains below 1.5 for windseas and below 1.1 for swell. Alpers and Brüning (1986) have shown that the stretching is caused mainly by velocity bunching, although the subresolution scale velocity spread $A_{v}$ also contributes. The statistical rms error of $S$ is of the order of 5-10 per cent.

The dependency of the stretching parameter. $S$ on the RAR MTF was investigated by additional SAR simulations for fixed $C^{\max }$ and $\Phi_{\mathrm{m}}$. The results are summarized in table 3. $S^{\min }$ and $S^{\max }$ represent the minimum and maximum stretching parameter, respectively, found within a sample of nine simulations obtained by combining the three values $|M|^{\text {RAR }}=5,10,15 \eta^{\text {RAR }}=0^{\circ}, 45^{\circ}, 90^{\circ}$. Typically, differences in $S$ up to 25 per cent occurred within a sample. However, no clear relationship emerged between the minimum (maximum) $S$ parameters and specific moduli and phases of the RAR MTF. Therefore an averaged stretching parameter $S$ was calculated as the best estimate of $S$ for different RAR MTF settings. For fixed $C^{\max }, S$ deviates less than 10 per cent from the stretching parameters shown in figure 7, so that these curves may be regarded as valid for a rather broad range of RAR MTF values.

When swell is imaged in the presence of a very low windsea, $A_{v}$ can become smaller than the (Phillips saturation) value given by equation (13) (see Alpers and Brüning 1986). To study the effect of velocity spreading, additional simulation runs were carried out in which $A_{v}$ was taken as half and twice the value given by equation (13). Doubling $A_{v}$ increased $S$ by $5-10$ per cent in the range $30 \leqslant \Phi_{\mathrm{m}} \leqslant 150^{\circ}$ and by 15-35 per cent for azimuthally travelling waves, $-30^{\circ} \leqslant \Phi_{\mathrm{m}} \leqslant 30^{\circ}$. Halving $A_{v}$

Table 3. Mean stretching parameter $S$ derived from the average of nine simulations with nine different RAR MTFs obtained by combining all values $\left|M^{\mathrm{RAR}}\right|=5,10,15$, and $\eta^{\mathrm{RAR}}=0^{\circ}$, $45^{\circ}, 90^{\circ}$ for fixed velocity bunching parameter $C^{\max }$ and azimuth angles $\Phi_{\mathrm{m}} . S^{\min }$ and $S^{\max }$ denote the smallest and largest $S$ parameter within a sample of nine experiments.

\begin{tabular}{lccll}
\hline$C^{\max }$ & $\Phi_{\mathrm{m}}$ & $S$ & $S^{\min }$ & $S^{\max }$ \\
\hline 2.3 & $30^{\circ}$ & 1.33 & 1.24 & 1.34 \\
$1 \cdot 1$ & $30^{\circ}$ & 1.08 & 1.0 & 1.24 \\
0.55 & $30^{\circ}$ & 0.92 & 0.84 & 1.07 \\
$2 \cdot 3$ & $60^{\circ}$ & 1.01 & $0.8 \ddagger$ & $1.14 \ddagger$ \\
$1 \cdot 1$ & $60^{\circ}$ & 0.91 & $0.8 \pm$ & $1.0 \ddagger$ \\
0.55 & $60^{\circ}$ & 0.88 & 0.73 & 0.95 \\
2.3 & $90^{\circ}$ & 0.81 & 0.77 & 0.96 \\
$1 \cdot 1$ & $90^{\circ}$ & 0.91 & 0.77 & 0.96 \\
0.55 & $90^{\circ}$ & 0.84 & 0.74 & 0.96 \\
2.3 & $120^{\circ}$ & 0.97 & 0.8 & 0.99 \\
$1 \cdot 1$ & $120^{\circ}$ & 0.91 & 0.8 & 0.99 \\
0.55 & $120^{\circ}$ & 0.91 & 0.89 & 0.99 \\
2.3 & $150^{\circ}$ & 1.34 & 1.24 & 1.44 \\
$1 \cdot 1$ & $150^{\circ}$ & 1.23 & 1.13 & 1.24 \\
0.55 & $150^{\circ}$ & 0.94 & 0.87 & 1.02 \\
\hline
\end{tabular}

tvalue representative for: $\left|M^{\mathrm{RAR}}\right|=10, \eta^{\mathrm{RAR}}=0^{\circ} ; \mid M^{\mathrm{RAR}}=15, \eta^{\mathrm{RAR}}=0^{\circ}, 45^{\circ}$.

fvalue representative for: $\left|M^{\mathrm{RAR}}\right|=5, \eta^{\mathrm{RAR}}=0^{\circ}, 45^{\circ}, 90^{\circ} ;\left|M^{\mathrm{RAR}}\right|=10, \eta^{\mathrm{RAR}}=0^{\circ}, 45^{\circ}, 90^{\circ}$. 
decreased $S$ by $5-10$ per cent for $30^{\circ} \leqslant \Phi_{\mathrm{m}}^{0} \leqslant 150^{\circ}$ and by $15-35$ per cent for $-30^{\circ} \leqslant \Phi_{\mathrm{m}}$ $\geqslant 30^{\circ}$. This supports the conclusion that stretching is produced primarily by velocity bunching rather than velocity spreading.

Alpers (1983) also found in his one-dimensional Monte-Carlo simulations that the SAR peak wavenumber is shifted in the nonlinear imaging regime towards lower wavenumber for azimuthally travelling waves. He predicted a rotation of the spectral peak in the two dimensional case towards the range direction. The shift and the rotation of the spectral peak has been observed by several authors in Seasat and SIR. B SAR ocean wave images (cf. Vesecky and Stewart 1982, Beal et al. 1983, Monaldo and Lyzenga 1986, Alpers et al. 1986, Brüning et al. 1988).

In the linear imaging regime, $S$ is the order unity or slightly smaller (figure 7). Values of $S<1$ arise because the MTF relating the wave spectrum $E(\mathbf{k})$ and the SAR image spectrum $F(\mathbf{k})$ is approximately proportional to $k^{n}$ where $2 \leqslant n \leqslant 3$, ( $n=2$ when the RAR MTF is dominant, and $n=3$ if velocity bunching is more important. Thus the peak of $F(\mathbf{k})$ is shifted to higher wavenumbers relative to the peak of $E(\mathbf{k})$.

It should be pointed out, however, that in computations of SAR image spectra for a global wave hindcast during the SEASAT period, Hasselmann et al. (1988) found no cases in which the purely linear theory, (without inclusion of an azimuthal cut-off factor) could be meaningfully applied to infer the spectral shape of the SAR spectrum. In all realistic cases, including weak swell spectra, the decay of the wave spectrum towards higher wave numbers was weaker than the $k^{3}$ increase of the velocity bunching MTF. This resulted in an unrealistic linear image spectrum which increased monotonically with $k$ without a discernible spectral peak. In practice, it appears that the purely linear theory can be applied only to the low wavenumber end of the spectrum and the position of the SAR spectral peak is generally determined by the transition from the linear to the nonlinear domain. This is confirmed by the recent analysis of Hasselmann and Hasselmann (1990). The authors find that the closed transformation relation yields as next order after the purely linear theory a quasilinear expression in which the linear SAR spectrum is simply multiplied by an azimuthal cut-off factor $\exp \left(-k_{x}^{2}\left\langle\xi^{2}\right\rangle\right)$.

\subsection{Rotation parameter}

The rotation of the spectral peak is shown in figure 8, again as a function of the velocity bunching parameter $C^{\max }$ and the propagation direction of the dominant ocean wave $\Phi_{\mathrm{m}}$, for fixed velocity spreading parameter $A_{v}=51$. The rotation is generally towards the range direction. It increases with $C^{\max }$ and is greatest for waves propagating in the azimuthal direction. As in the case of the stretching parameter, the rotation is caused primarily by the nonlinearity of the velocity bunching mechanism, although the sub-resolution scale velocity spread also contributes (see Alpers and Brüning 1986).

To investigate the dependence of the rotation parameter $\Delta \Phi$ on the RAR MTF, additional SAR simulations were carried out for the nine cases listed previously with $\left|M^{\text {RAR }}\right|=5,10,15$ and $\eta^{\text {RAR }}=0^{\circ}, 45^{\circ}, 90^{\circ}$. The results are presented in figure $9(a)-(e)$, together with the core simulation curves from figure 8 for fixed azimuth angles $\Phi_{\mathrm{m}}$. The curves show $\Delta \Phi$ for different RAR MTF settings for fixed values of the velocity bunching parameter $C^{\max }\left(C^{\max }=0 \cdot 55,1 \cdot 1,2 \cdot 3\right)$. In figures $9(a)$, (e), $\left(\Phi_{\mathrm{m}}=30^{\circ}, 150^{\circ}\right)$ the deviations from the core simulations (dotted lines) are generally smaller than $10^{\circ}$ (except for very large moduli of $M^{\text {RAR }}$ ). We infer from these 


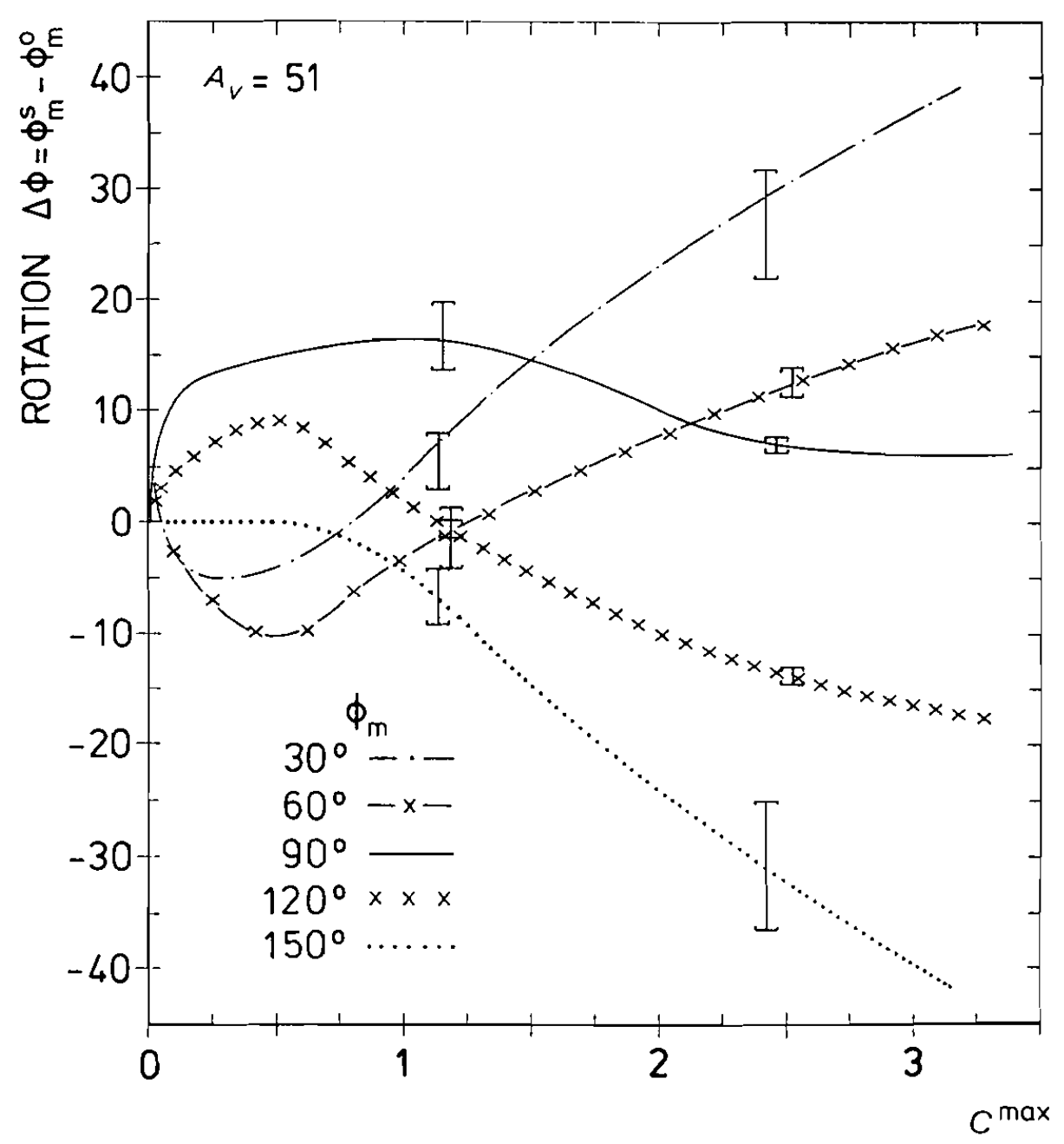

Figure 8. Rotation of the spectral peak as a function of the nonlinearity parameter $C^{\max }$ for different azimuth angles $\Phi_{\mathrm{m}}\left(A_{v}=51\right)$.

figures that the dependence of the rotation parameter on the RAR MTF is small as long as the dominant ocean wave direction lies fairly close to the azimuthal direction $\left(\Phi_{\mathrm{m}}=0^{\circ}, 180^{\circ}\right)$. However, as the propagation direction of the dominant ocean wave approaches the range direction $\left(\Phi_{\mathrm{m}}=90^{\circ}, 270^{\circ}\right)$, the velocity bunching mechanism becomes weaker and the SAR imaging process is more strongly affected by variations of the RAR MTF. This can be seen from figures $9(b)-(c)\left(\Phi_{\mathrm{m}}=60^{\circ}, 90^{\circ}, 120^{\circ}\right)$. In this parameter regime, deviations of $\Delta \Phi$ from the core simulation values can exceed $30^{\circ}$. The occasional jumps in $\Delta \Phi$ are due to an interchange of the primary and secondary peaks seen on either side of the range wavenumber axis in figures 5 and $6((b)$ and (b)-(d), respectively).

The dependence on $A_{v}$ is weak. Simulation runs in the strongly nonlinear imaging regime $\left(C^{\max }>1.5\right)$ with a doubled velocity spread parameter $A_{v}=100$ yielded an 
(a)

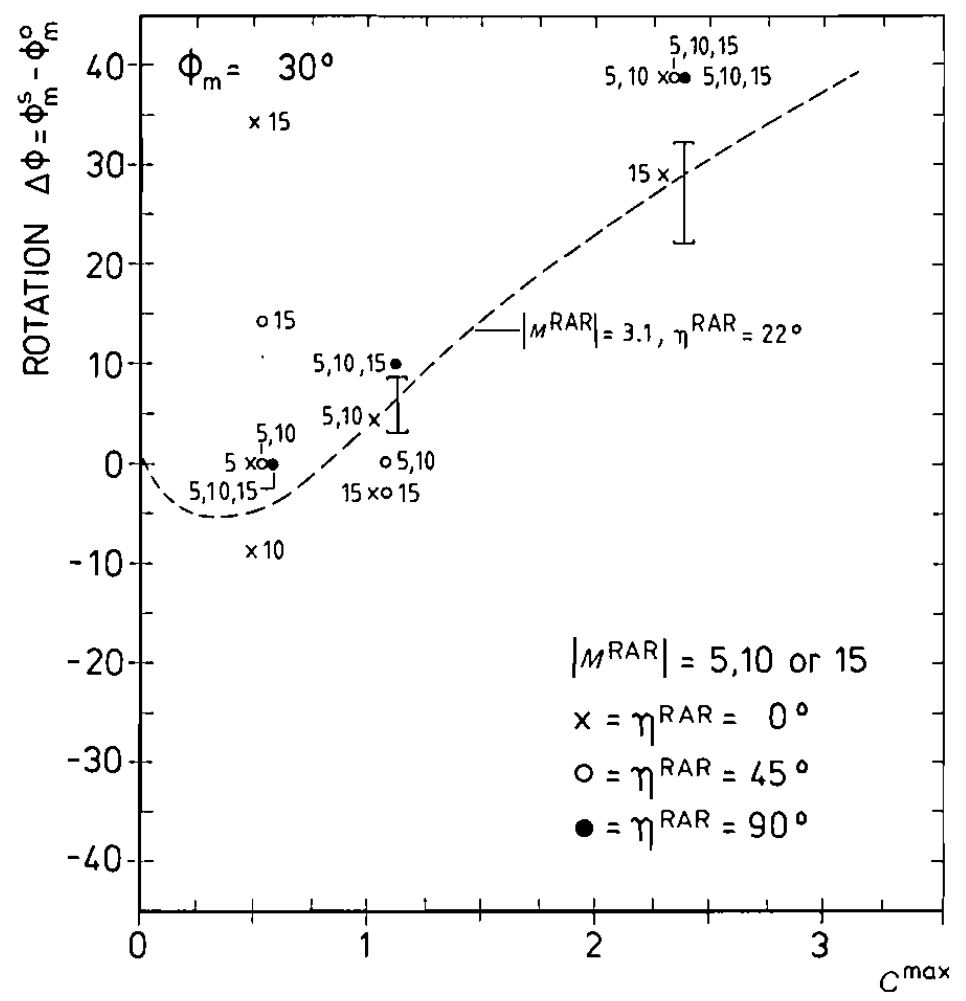

(b)

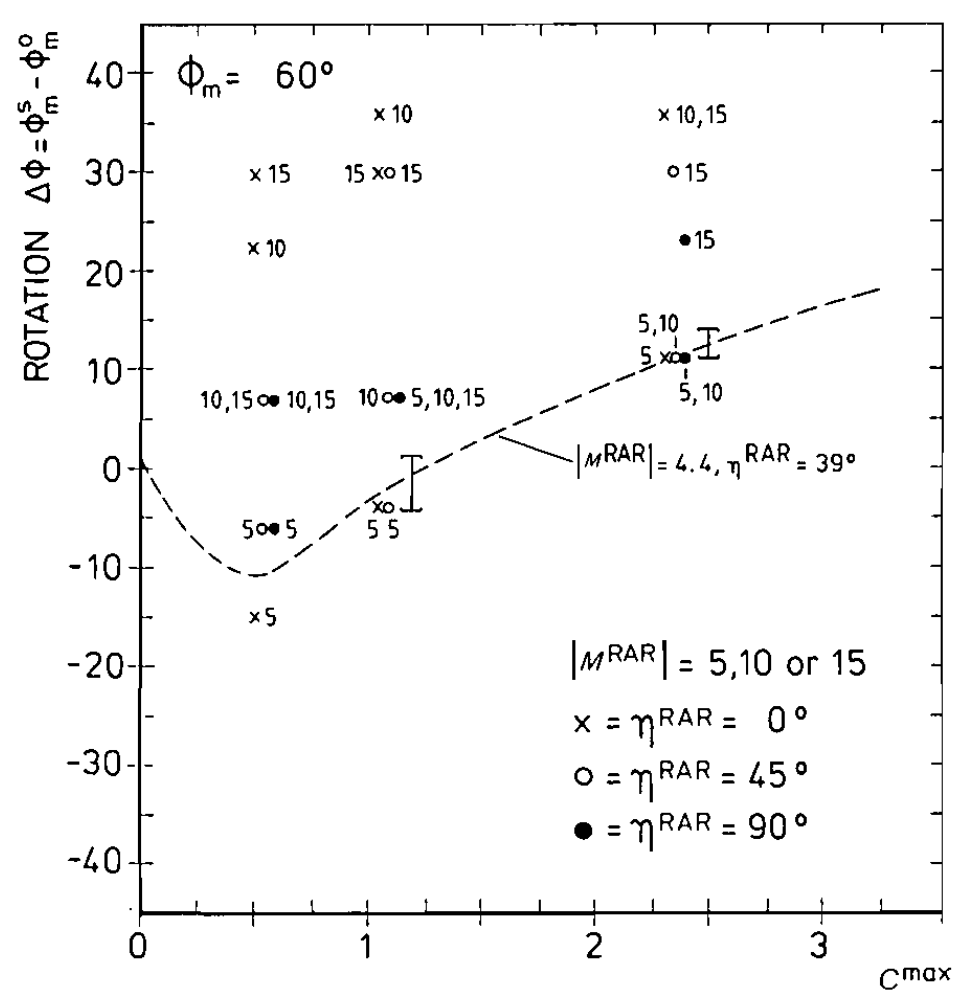


Simulation of SAR imaging by ocean waves

(c)

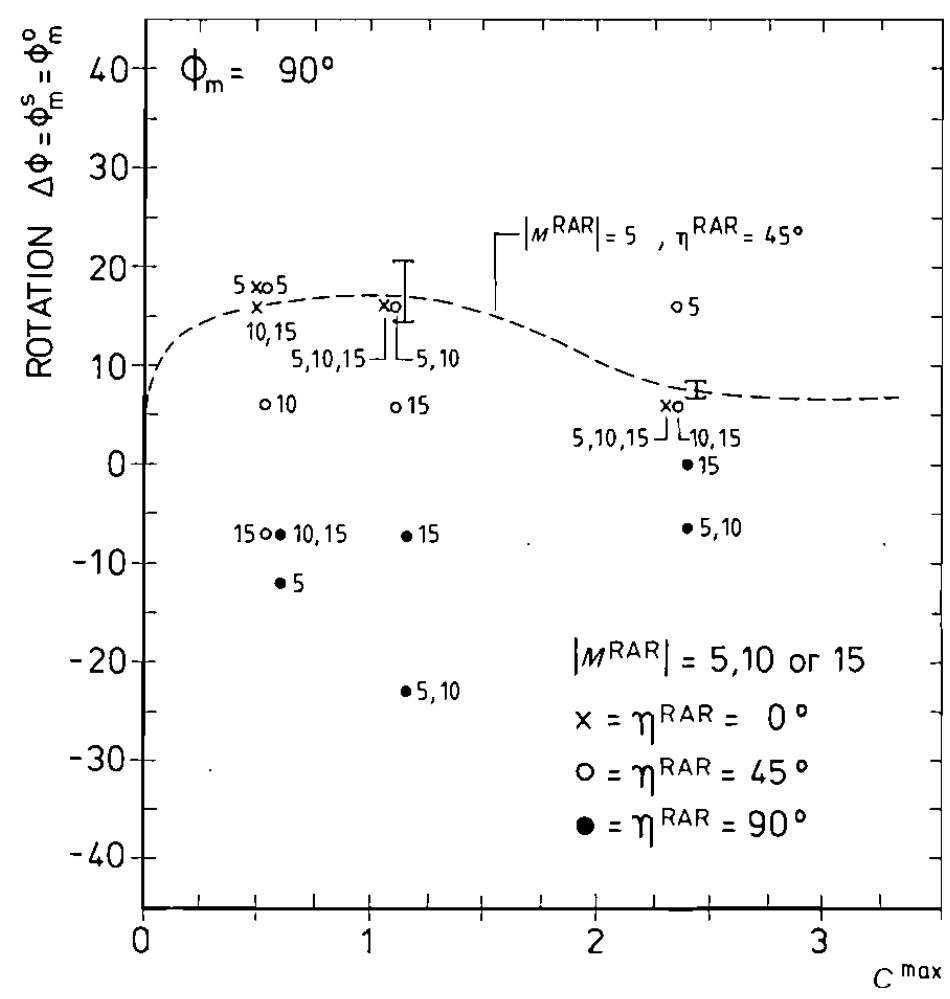

(d)

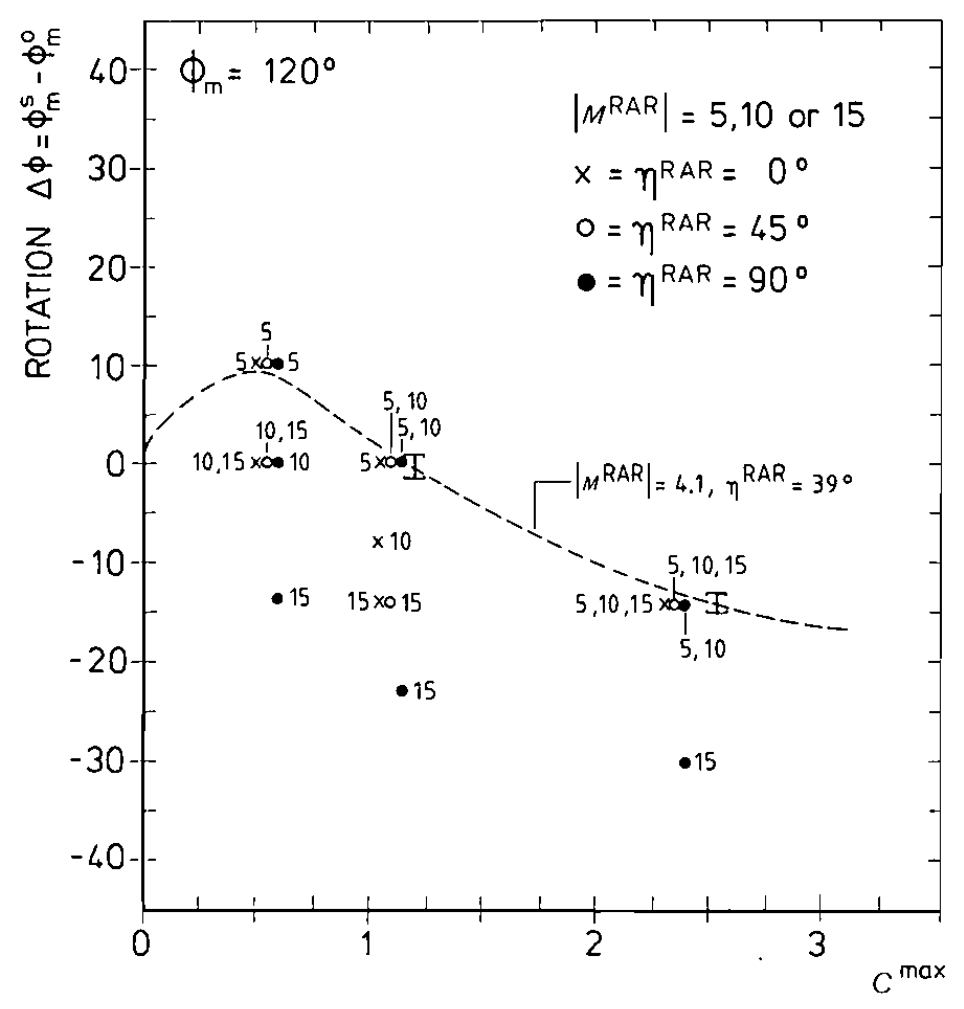


$(e)$

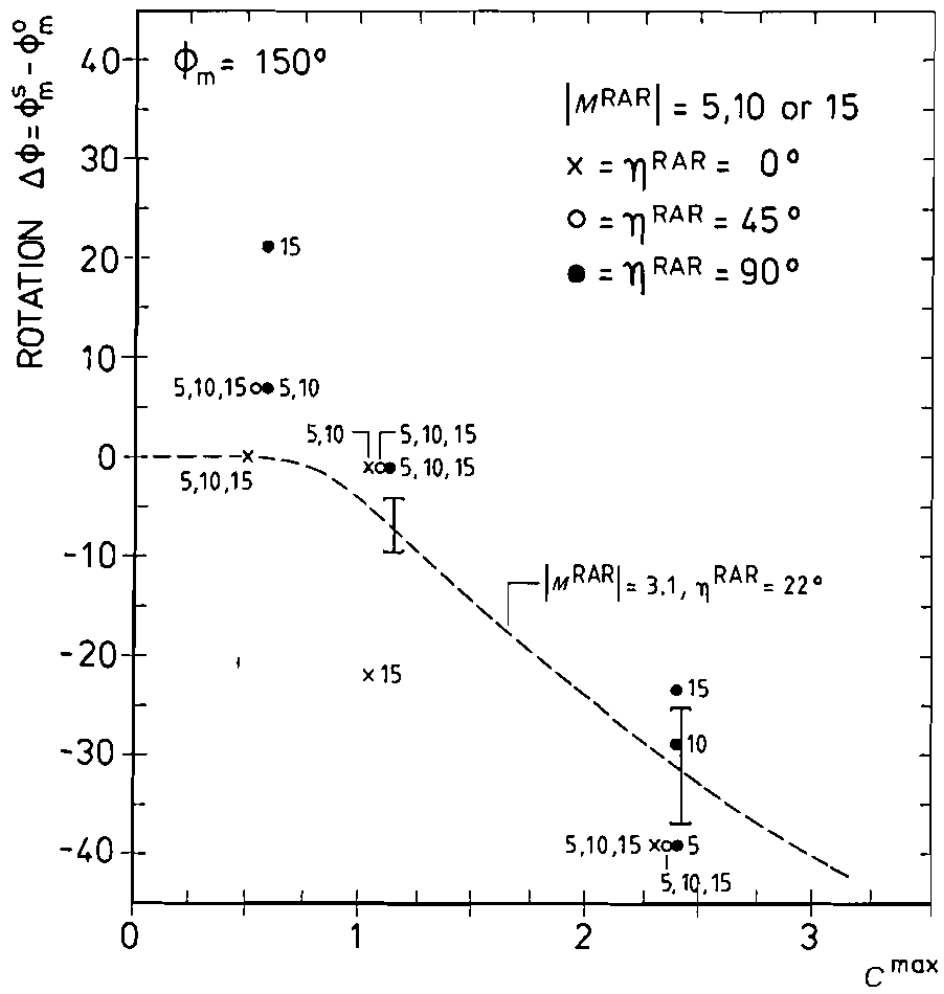

Figure 9. Rotation $\Delta \Phi$ of the spectral peak as a function of the nonlinearity parameter $C^{\max }$ for different moduli and phases of the RAR MTF and for fixed azimuth angles $(a)$, $\Phi_{\mathrm{m}}=30^{\circ} ;(b), \Phi_{\mathrm{m}}=60^{\circ} ;(c), \Phi_{\mathrm{m}}=90^{\circ} ;(d), \Phi_{\mathrm{m}}=120^{\circ} ;(e) ; \Phi_{\mathrm{m}}=150^{\circ}$. Also shown as dotted curves are the core simulation results from figure 8 for particular azimuth.

increased rotation by $8^{\circ}$ to $12^{\circ}$. Halving $A_{v}$ to 25 decreases $\Delta \Phi$ by a similar amount. The rotation angle showed some dependence on the shape of the spectrum, narrowpeak swells with a narrow directional distribution exhibiting smaller rotation angles.

In the linear imaging regime $\left(C^{\max }<1\right)$, figure 8 occasionally shows a rotation of the peak wavenumber towards the azimuthal direction. This results from the interference of the RAR and velocity bunching modulation transfer functions. The magnitude of the rotation can be as large as $15^{\circ}$ for windseas but is generally smaller than $3^{\circ}$ for swell (this is of the order of the $\Delta \Phi$ r.m.s. error indicated in figure 8 ).

\subsection{Signal-to-clutter ratio}

The signal-to-clutter ratio $(\mathrm{S} / \mathrm{C})$ is shown in figure 10 , in the same format as figures 7 and 8 , as a function of $C^{\max }$ and $\Delta \Phi$, for fixed $A_{v}=51$. The upper abscissa axis shows the corresponding values for $B=\left(k_{\mathrm{m}} \rho_{\mathrm{a}}\right)^{-1}$ (cf. equation (17)). The variation of $C^{\max }$ and $B$ in this figure should be interpreted as before as due to changes in the fully developed sea state, i.e. the peak wavenumber or wind speed, for fixed SAR parameters (the $B$ values correspond to Seasat SAR parameter values). In the medium and strongly nonlinear imaging regime $\left(C^{\max }>1 \cdot 5\right), \mathrm{S} / \mathrm{C}$ is largest for waves propagating in the range direction and smallest for waves propagating in the azimuthal direction. It increases with decreasing $C^{\max }$ (increasing $B$ ). For azimuthally 


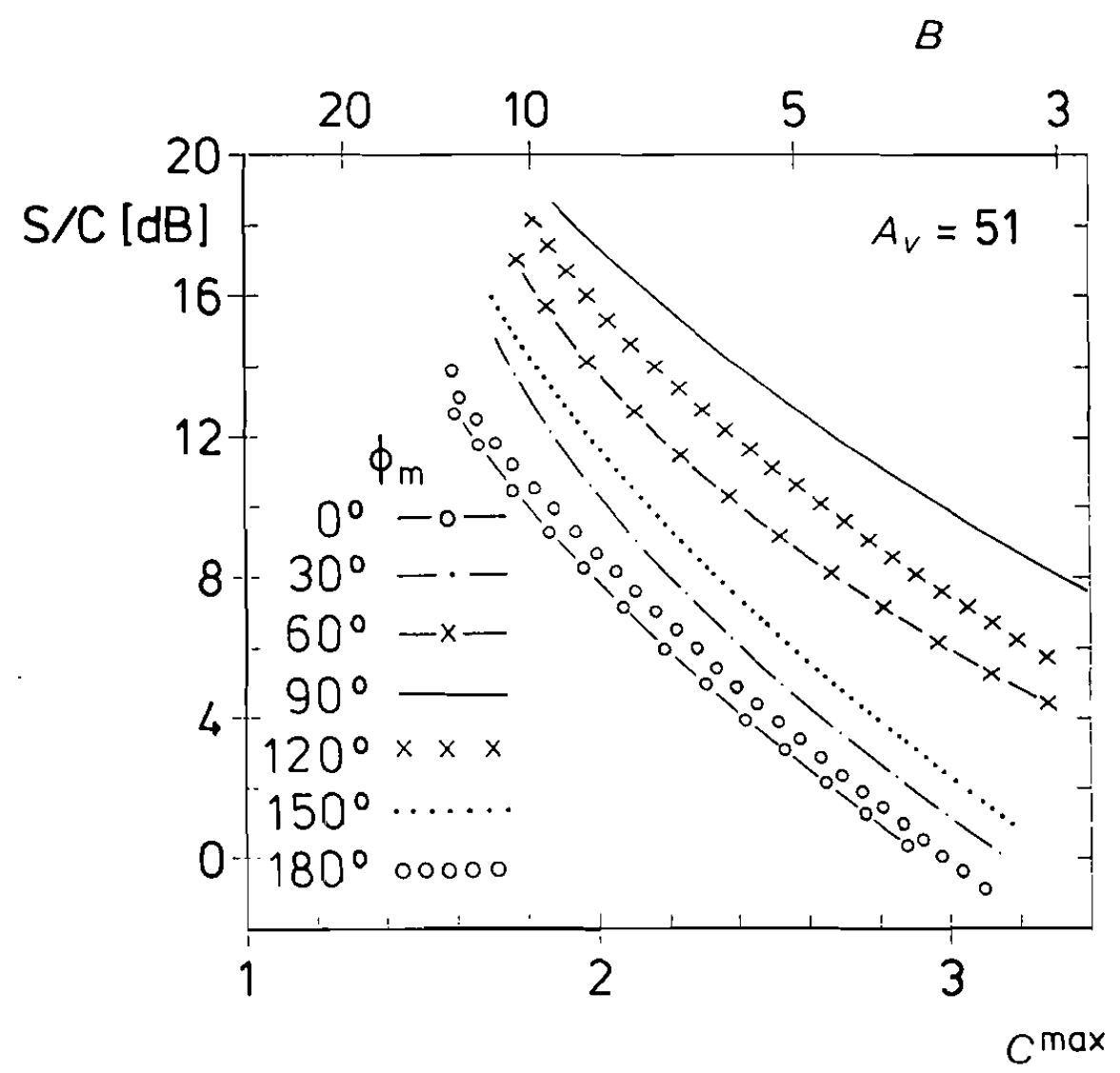

Figure 10. Spectral signal-to-clutter ratio (S/C) as a function of the nonlinearity parameter $C^{\max }$ or $B$ for different azimuth angles $\Phi_{\mathrm{m}}\left(A_{v}=51\right)$. The relation between $C^{\max }$ and $B$ is based on Seasat SAR parameter values.

travelling waves, the ocean wave signal disappears in the clutter noise $(\log [\mathrm{S} / \mathrm{C}]<0)$ for $C^{\max }>3(B<4)$.

In contrast to the stretching parameter and the directional rotation, the signal-toclutter ratio shows a rather strong dependence on $A_{v}$. Simulations with $A_{v}=100$, indicate that azimuthally travelling waves become undetectable already for $C^{\max }>2$, $B<8$. (However, the effect of $A_{v}$ on the signal-to-clutter ratio is better expressed in terms of the detection wave length cut-off, cf. following subsection.)

Figure 11 shows $\mathrm{S} / \mathrm{C}$ as a function of $C^{\max }$ and $\Phi_{\mathrm{m}}$ for fixed $B(B=5 \cdot 6)$. This figure may be interpreted as keeping the sea state and SAR resolution fixed while varying $R / V$. The corresponding values of $A_{v}$ are shown on the upper abscissa axis. The signal-to-noise ratio first increases with increasing $C^{\max }$ but then decreases again as the velocity bunching parameter moves into the nonlinear regime. In the linear regime, the interference of the RAR and velocity bunching modulation produces variations in $\mathrm{S} / \mathrm{C}$ for waves travelling in different propagation directions of the order of 3-6 dB. The curves for different values of $B$ are similar, the main effect of increasing $B$ being to increase $\mathrm{S} / \mathrm{C}$ by a comparable factor (increasing $B$ by a factor of 


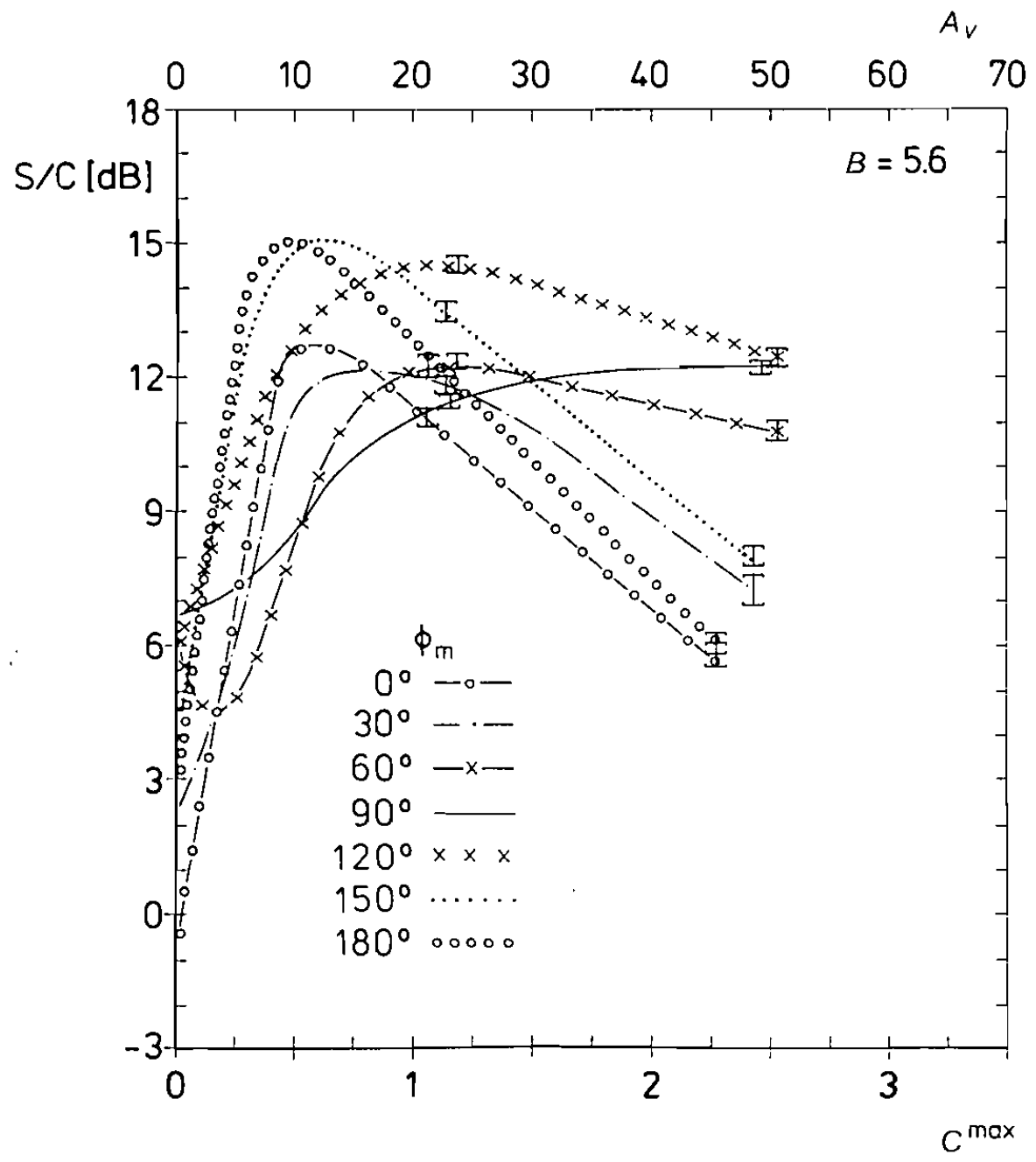

Figure 11. Spectral signal-to-clutter ratio $(\mathrm{S} / \mathrm{C})$ as a function of the nonlinearity parameter $C^{\max }$ for different azimuth angles $\Phi_{\mathrm{m}}$ and fixed $B=5 \cdot 6$.

two increases $\mathrm{S} / \mathrm{C}$ by about $6 \mathrm{~dB}$. The $\mathrm{S} / \mathrm{C}$ r.m.s error values in these calculations were less than $\pm 1.0 \mathrm{~dB}$, cf. figure 11 ).

Simulation experiments were also carried out to test the dependence of the imaging mechanism on the modulus and phase of the RAR MTF. Only a weak dependence on $\left|M^{\text {RAR }}\right|$ was found. In the linear and weakly nonlinear imaging regimes, $\mathrm{S} / \mathrm{C}$ decreases by $1-2 \mathrm{~dB}$ for an increase of $\left|M^{\text {RAR }}\right|$ by a factor of two (to $\left.\left|M^{\text {RAR }}\right|=10\right)$. Still smaller changes are found in the strongly nonlinear regime. Somewhat more sensitivity was found with respect to the phase $\eta^{\text {RAR }}$ of the RAR modulation transfer function. In the simulation runs shown in figure $12, \eta^{\text {RAR }}$ was varied in the range $-90^{\circ}<\eta^{\mathrm{RAR}}<90^{\circ}$ in steps of $30^{\circ}$, with fixed $\left|M^{\mathrm{RAR}}\right|=5$ and $C^{\max }$ values of 0.5 (figure 12a)), 1.1 (figure $12 b$ )) and 2.3 (figure $12(c)$ ). Measurements of $\eta^{\text {RAR }}$ (Wright et al. 1980, Schroeter et al. 1986, Feindt 1985) generally yield values in the range $\pm 45^{\circ}$, depending on wind speed, ocean wave propagation direction and 
(a)

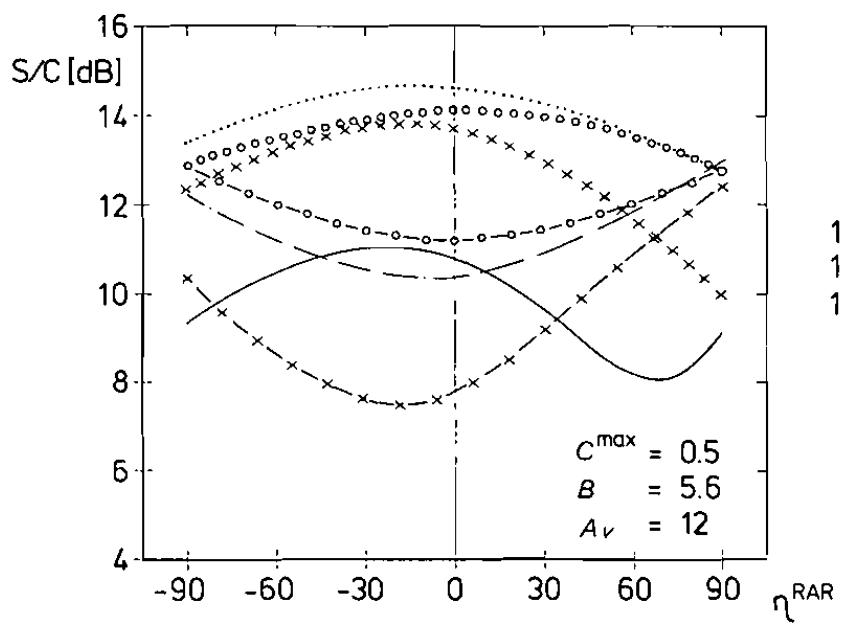

(b)

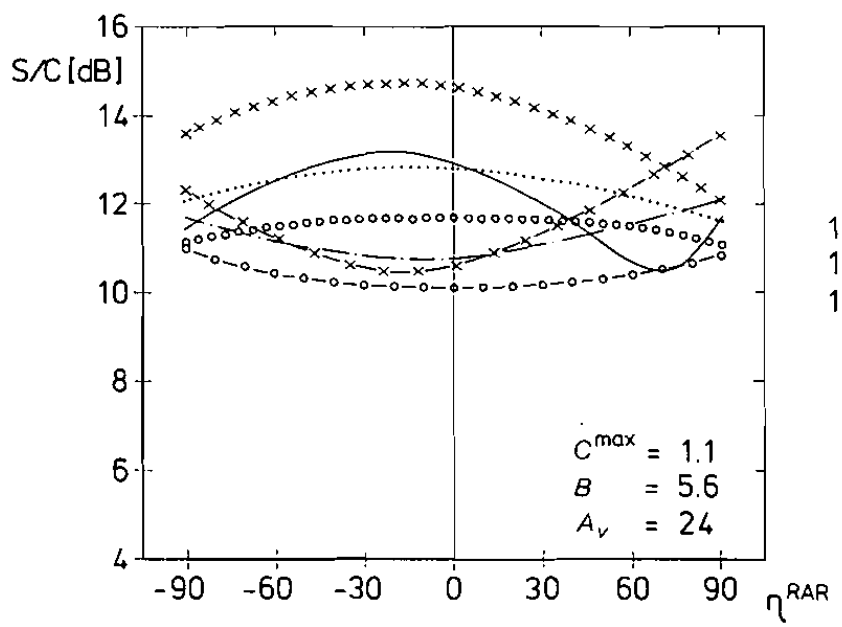

$(c)$

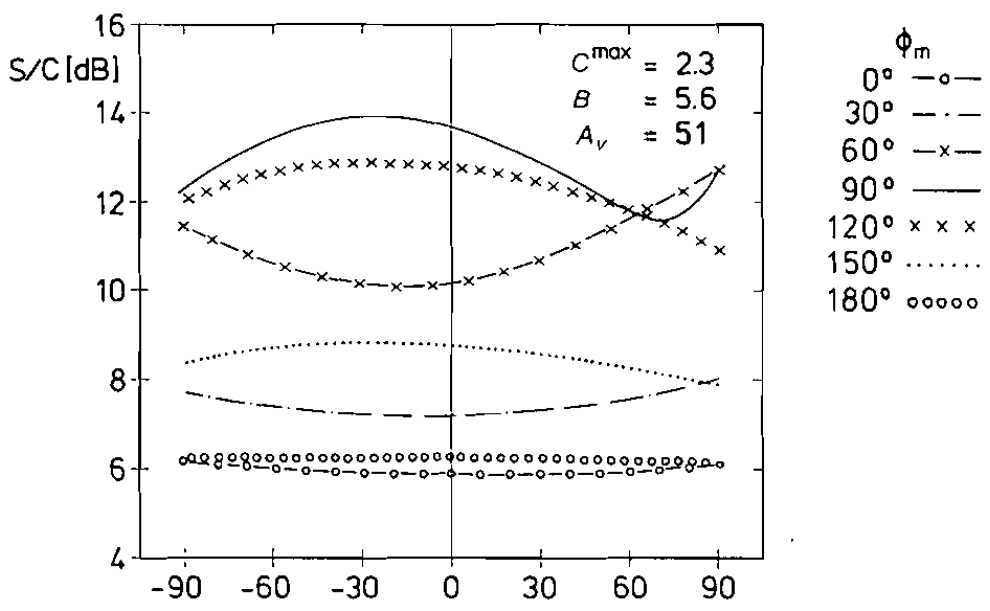

Figure 12. Spectral signal-to-clutter ratio $(\mathrm{S} / \mathrm{C})$ as a function of the phase $\eta^{\mathrm{RAR}}$ of the RAR MTF. (a) Linear imaging regime $C^{\max } \approx 0 \cdot 5, A_{v}=11 \cdot 9$, and $\mathrm{B}=5 \cdot 6 ;(b)$ weakly nonlinear, $C^{\max } \approx 1.1$ and $A_{v}=23 \cdot 5$; $(c)$ strongly nonlinear, $C^{\max } \approx 2.3$ and $A_{v}=51$. The value of the modulus of the RAR MFT is 5 . 
other parameters. In the linear imaging regime $\left(C^{\max }=0 \cdot 5\right)$, the variation of the signal-to-clutter ratio due to interference effects can exceed $5 \mathrm{~dB}$ if the cross-section and velocity bunching MTFs are of the same order of magnitude. However, in the strongly nonlinear regime dominated by velocity bunching $\left(C^{\max }=2 \cdot 3\right)$, the variation in $S / C$ is less than $1 \mathrm{~dB}$.

\subsection{Azimuthal cut-off wavelength}

The sub-resolution scale velocity spread smears the SAR image in the azimuthal direction and acts as an azimuthal low-pass filter. For the Seasat SAR the effective azimuthal cut-off wavelength has been estimated by Tucker (1983) to be of the order of $330 \mathrm{~m}$. This result is confirmed by our simulation studies. The non-dimensional azimuthal detection cut-off wavelength $\lambda_{\mathrm{cl}}^{\mathrm{d}}$ is approximately proportional to the azimuthal velocity smear parameter $A_{v}$ (cf. figure 13). To first order, $\lambda_{\mathrm{cl}}^{\mathrm{d}}$ is independent of the azimuth angle $\Phi_{\mathrm{m}}$ and the nonlinearity parameter $C^{\max }$ for windsea imaging. The error bars shown in figure 13 encompass the azimuthal fall-off wavelengths of all azimuth angles. However, $\lambda_{\mathrm{c}}^{d}$ shows a weak dependence on $C^{\max }$ for swell. Variations of $M^{\text {RAR }}$ affect $\lambda_{\text {cl }}^{\mathrm{d}}$ only marginally.

The degradation of the azimuthal resolution due to velocity spread also affects the detectability of the longer waves. Figure 14 shows polar plot isolines of the non-

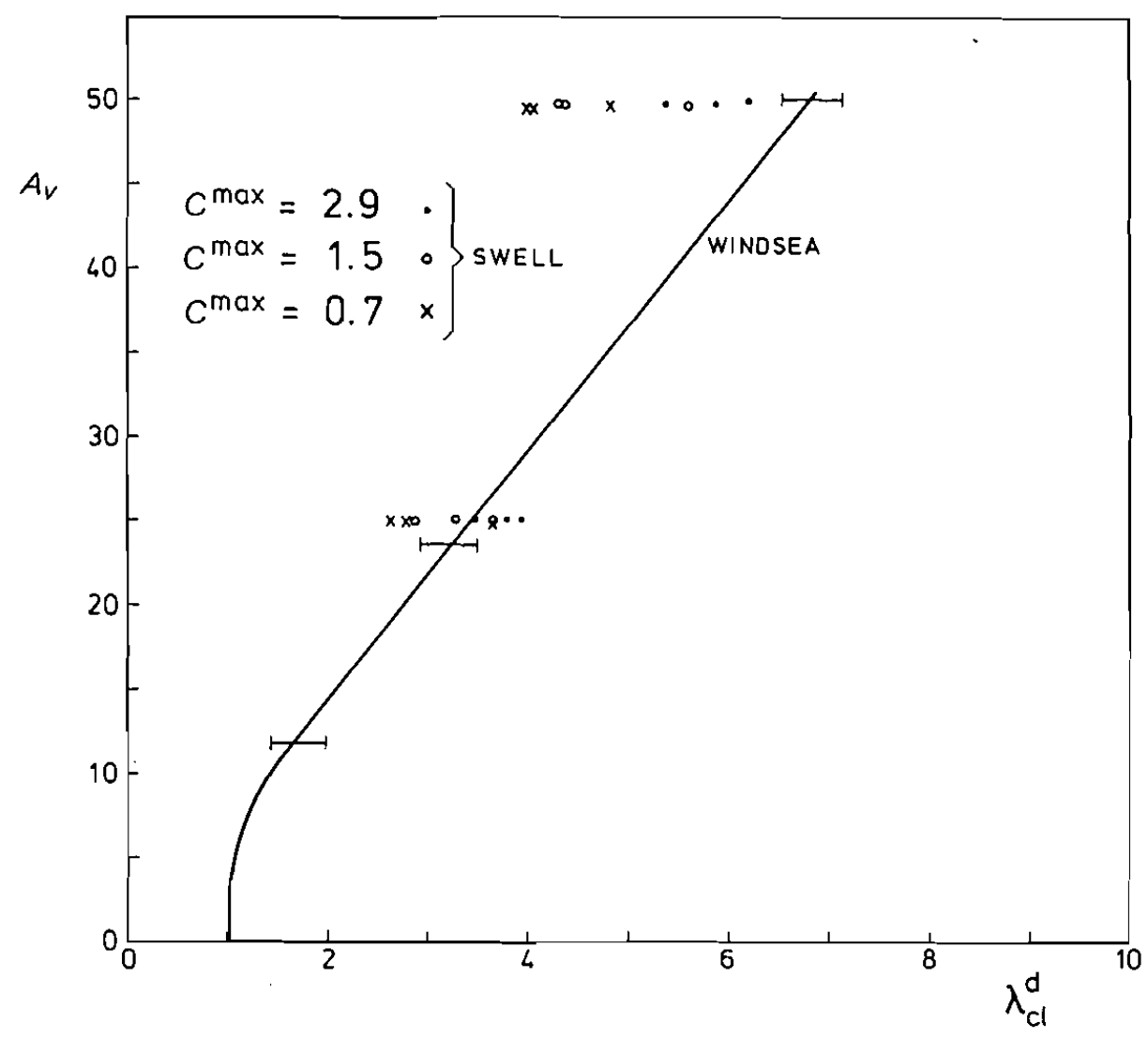

Figure 13. Non-dimensional azimuthal clutter detection cut-off wavelength $\lambda_{c l}^{d}$ as a function of $A_{v}$ for windsea and swell imaging. 


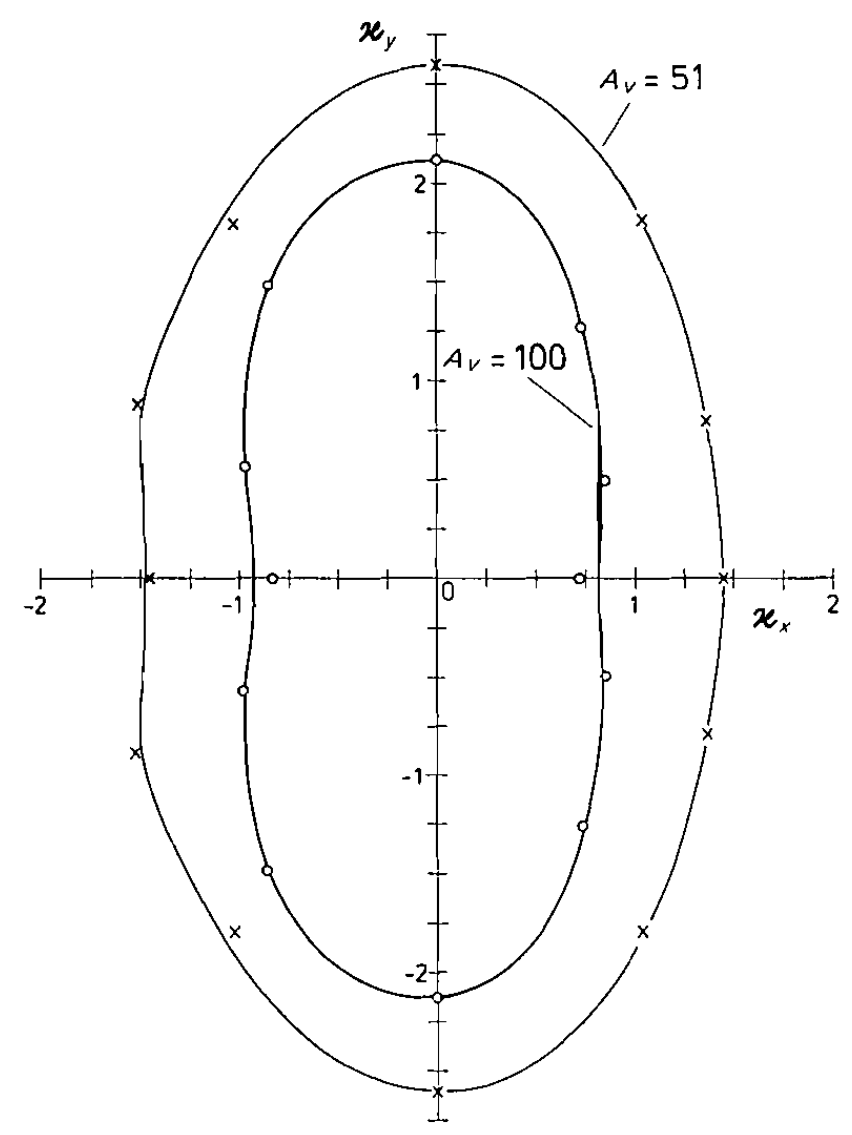

Figure 14. Isolines of the non-dimensional detectable ocean peak wave numbers $\kappa$ for fully developed windseas, for $A_{v}=51$ and $A_{v}=100$.

dimensional ocean peak wavenumber detection threshold $\kappa$ for a fully developed windsea for $A_{v}=51$ and 100. The components of $\kappa$ are defined as $\kappa_{x}=k_{\mathrm{m}}^{\mathrm{d}} \rho_{\mathrm{a}} N$, $\kappa_{y}=k_{\mathrm{m}}^{\mathrm{d}} \rho_{y}$, where $\rho_{\mathrm{a}} N$ and $\rho_{y}$ are the resolution scales in the azimuthal $(N$-look) and range directions, respectively, and $k_{\mathrm{m}}^{\mathrm{d}}$ is the marginally detectable peak wavenumber for which $\log [\mathrm{S} / \mathrm{C}]=0$. No waves at all are visible in the SAR image for peak wavenumbers $k_{\mathrm{m}}>k_{\mathrm{m}}^{\mathrm{d}}$. In computing figure 14, the SAR parameters were kept fixed at the Seasat values, $\mathrm{S} / \mathrm{C}$ varying only through $k_{\mathrm{m}}$ and $\Phi_{\mathrm{m}}$. Thus the loss of detectability is the combined result of the azimuthal cut-off and the reduction of $\mathrm{S} / \mathrm{C}$ with increasing $C^{\max }$. The weak asymmetry about the look direction is caused by the interference effects discussed earlier.

\section{Summary and conclusions}

The Monte-Carlo simulations carried out in this study demonstrate that the motions of the backscattering facets advected by the orbital velocity field of the long ocean waves have a strong affect on the SAR imaging of two-dimensional wave spectra. Nonlinear distortions occur for a wide range of ocean wave and SAR parameters. The principal effects can be expressed as a stretching of the peak 
wavelength, a rotation of the spectral peak towards the range direction, and an azimuthal spectral cut-off. All of these effects have been observed in SAR images.

The principal parameters governing the nonlinearity of the SAR imaging are the velocity bunching parameter $C^{\max }$ (equations (12)), and velocity spread parameter $A_{v}$ (equation (13)). These depend on non-dimensional combinations of SAR and sea state parameters. In addition, the imaging is strongly dependent on the mean ocean wave propagation direction $\Phi_{m}$ relative to the satellite flight direction. Strongly nonlinear distortions occur in the main part of the spectrum (large stretching parameters and rotation) in the parameter range $C^{\max } \cos \Phi_{\mathrm{m}}>2$. The signal to clutter ratio $(\mathrm{S} / \mathrm{C})$ is also reduced in this parameter range. The velocity spread parameter $\boldsymbol{A}_{v}$ affects mainly the high wavenumber azimuthal cut-off. The influence of the large scale radial orbital acceleration is generally smaller than the velocity bunching or velocity spread terms (see also Alpers and Brüning 1986).

The response of the SAR imaging process due to variations of the RAR MTF is small for azimuthally propagating ocean waves. As to be expected, it becomes stronger as the velocity bunching mechanism decreases for ocean waves propagating more into the range direction.

For wave spectra with a peak in the range direction, the spectrum generally splits into a double peaked SAR image spectrum. This is due to the vanishing of the velocity bunching mechanism for range travelling waves. Due to the interference of the velocity bunching and RAR modulation, the double peaks can be rather nonsymmetric with respect to the range direction, the details depending strongly on the modulus and phase of the RAR MTF. A more detailed discussion of these effects can be found in Brüning et al. 1988.

For the practical application of SAR wave imaging to wave forecasting and wave climate statistics, the inverse problem of converting the measured SAR spectrum to a wave spectrum will need to be addressed. This will presumably require multiple forward transformation computations in some iterative optimization scheme. For this and other problems, some form of parameterization would be desirable in which the principle feature of the transformation can be rapidly determined from a set of look-up tables. It is hoped that the comprehensive set of computations presented in this paper will provide a useful data base for developing such parameterization and inversion schemes in preparation for future applications of satellite SAR wave data.

It may be anticipated that future computations of SAR image spectra from ocean wave spectra and the development of inverse methods will be based on the computationally more efficient closed spectral transformation relations of Hasselmann and Hasselmann (1990). However, the Monte-Carlo technique applied in this paper will remain a useful tool, as it can readily be expanded to more general cases involving, for example, non-Bragg scattering processes.

\section{Acknowledgments}

This work was supported by the Bundesministerium für Forschung und Technologie, Bonn (BMFT 01 QS 86174), and by the Office of Naval Research, Washington (grant N 000 14-83-G-0126, monitored by H. Dolezalek).

\section{References}

Alpers, W., 1983, Monte Carlo simulations for studying the relationship between ocean wave and synthetic aperture radar image spectra. Journal of Geophysical Research, 88, 1745-1759. 
ALPERS, W., and BRÜNING, C., 1986, On the relative importance of motion-related contributions to the SAR imaging mechanism of ocean surface waves. I.E.E.E. Transactions on Geoscience and Remote Sensing, 24, 873-885.

Alpers, W., BrüNiNG, C., and RICHTER, K., 1986, Comparison of simulated and measured synthetic aperture radar image spectra with buoy-derived ocean wave spectra during the Shuttle Imaging Radar-B mission, I.E.E.E. Transactions on Geoscience and Remote Sensing. 24, 559-566.

ALPERS, W., and HASSELMANN, K., 1982, Spectral signal to clutter and thermal noise properties of ocean wave imaging synthetic aperture radars. International Journal of Remote Sensing. 3, 423-446.

Alpers, W., Ross, D. B., and RufenaCH, C. L., 1981, On the detectability of ocean surface waves by real and synthetic aperture radar. Journal of Geophysical Research, 86, 6481.

Alpers, W., and RufENaCH, C. L., 1979, The effect of orbital motions on synthetic aperture radar imagery of ocean waves. I.E.E.E. Transactions on Antennas and Propagation, 27, 685-690.

Bass, F. G., Fuks, I. M., Kalinowsky, A. I., Ostrowsky, I. E., and RosenberG, A. D., 1968, Very high frequency radio wave scattering by a disturbed sea surface. I.E.E.E. Transactions on Antennas and Propagation, 16, 554-568.

BEal, R. C., Tilly, D. G., and Monaldo, F. M., 1983, Large- and small scale spatial evolution of digitally processed ocean wave spectra from Seasat synthetic aperture radar. Journal of Geophysical Research, 88, 1761-1778.

Brüning, C., Alpers, W., Zambresky, L. F., and Tilley, D. G., 1988, Validation of a SAR ocean wave imaging theory by the Shuttle Imaging Radar-B experiment over the North Sea. Journal of Geophysical Research, 93, 15403-15425.

Chandry, A. H., Gogineni, S. P., and MOORE, R. K., 1986, Tower-based broadband backscattering measurements from the ocean surface in the North Sea. Proceedings of the International Geoscience and Remote Sensing Symposium (IGARSS '86) held in Zurich, Switzerland, on 8-11 September 1986, Vol. 1, ESA SP-24 (Noordwijk: European Publication Division, ESTEC), pp. 327-335.

Elachi, C. E., and Brown, W. E., 1977, Models of radar imagery of ocean surface waves. I.E.E.E. Transactions on Antennas and Propagation, 25, 84-95.

FeINDT, F., 1985, Radar-Rueckstreuexperimente am Wind-Wellen-Kanal bei sauberer und filmbedeckter Wasseroberflaeche im X-Band $(9 \cdot 8 \mathrm{GHz})$. Hamburger Geophysikalische Einzelschriften, Heft 75, University of Hamburg.

Feindt, F., Schroeter, J., and Alpers, W., 1986, Measurements of the occan wave radar modulation transfer function at $35 \mathrm{GHz}$ from a sea-based platform in the North Sea. Journal of Geophysical Research, 91, 9701-9708.

HARGER. R. O., 1980, The synthetic aperture radar image of time variant scenes. Radio Science, $15,749-757$.

Hasselmann, K., Barnett, T. P., Bouws, E., Carlson, H., Cartwright, D. E., Enke, K., Ewing, J. A., Gienapp, H., Hasselmann, D. E., Kruseman, P., Meerburg, A., Mueller, P., Olbers, D. J., Richter, K., Sell, W., and Walden, H., 1973, Measurements of wind-wave growth and swell decay during the Joint North Sea Wave Project (JONSWAP). Deutsche Hydrographische Zeitschrift, Reihe A, No. 12, pp. 1-95.

HasselmanN, D.E., DunCkel, M. and EwING, J. A., 1980, Directional wave spectra observed during JONSWAP, 1973. Journal of Physical Oceanography, 10, 1264-1280.

HasselmanN, K., and HasselmanN, S., 1990, On the nonlinear mapping of an ocean wave spectrum into a SAR image spectrum and its inversion. Max-Planck-Institute for Meteorology, Hamburg.

Hasselmann, K.. Hasselmann, S., Bauer, E., Brüning, C., Lehner, S., Graber, H., and LIONEllo P., 1988, Satellite SAR image spectra and altimeter wave height data assimilation system for ERS-1. Final Report, (European Space Agency Study Contract No. 6875/87/HGE-I(SC). Max-Planck-Institute Report No. 19.

Hasselmann, K., Hasselmann, S., Brüning, K., and Speidel, A., 1990, Application of a new closed ocean wave-SAR spectral transform relation to LEWEX data. Proceedings of the Symposium on Directional Ocean Wave Spectra held in Laurel, Maryland, on 18-20 April, 1989, Johns Hopkins APL Technical Digest (in the press).

Hasselmann, K., Raney, R. K., Plant, W. J., Alpers, W., Shuchman, R. A., Lyzenga, D. R.. RufenaCh, C. L., and TuCKer, M. J., 1985, Theory of synthetic aperture radar ocean imaging: A MARSEN View. Journal of Geophysical Research, 90, 4659-4685. 
HolthuiJsen, L. H., 1981, The directional distribution of wind generated waves as inferred from stereo-photographic observations of the sea surface. Communication on Hydraulics, Department of Civil Engineering, Delft University of Technology, The Netherlands, Report No. 81-2.

IVANOv, A. V., 1983, On the mechanism of imaging ocean waves by synthetic aperture radar. I.E.E.E. Transactions on Antennas and Propagation, 31, 538-541.

LYZENGA, D. R., 1986, Numerical simulation of synthetic aperture radar image spectra for ocean waves. I.E.E.E Transactions on Geoscience and Remote Sensing, 24, 863-872.

Mitsuyasu, H., Tasai, F., Suhara, T., Mizuno, S., Ohkusu, M., Honda, T., and Rikischi, K., 1975, Observations of the directional spectrum of ocean wave using a clover leaf buoy. Journal of Physical Oceanography, 5, 750-760.

MoNAldO, R., 1984, Improvement in the estimation of dominant wave number and direction from spaceborne SAR image spectra when corrected for ocean surface movement. I.E.E.E Transactions on Geoscience and Remote Sensing, 22, 603-608.

Monaldo, F. M., and LyzenGa, D. R., 1986, On the estimation of wave slope- and heightvariance spectra from SAR imagery. I.E.E.E. Transactions on Geoscience and Remote Sensing, 24, 543-551.

Ouch!, K., 1984. Two-dimensional imaging mechanisms of ocean waves by synthetic aperture radars. Journal of Physics D, 17, 25-42.

PhILlips, O., 1977, Dynamics of the Upper Ocean, 2nd edn (Cambridge: Cambridge University Press).

Plant, W. J., and Keller, W. C., 1983, The two-scale radar wave probe and SAR imagery of the ocean. Journal of Geophysical Research, 88, 9776-9784.

RANEY, R. K., 1980, SAR response to partially coherent phenomena. I.E.E.E. Transactions on Antennas and Propagation, 28, 777-787.

RANEY, R. K., 1981, Wave orbital velocity, fade and SAR response to azimuth waves. Journal of Oceanic Engineering, 6, 140-146.

RANEY, R. K., and VACHON, P. V., 1988, Synthetic aperture radar imaging of ocean waves from an airborne platform focus and tracking issues. Journal of Geophysical Research, 93, $12475-12486$.

Rothleram, S., 1983, Theory of SAR ocean wave imaging. In Satellite Microwave Remote Sensing, edited by T. D. Allan (Chichester: Ellis Horwood Ltd.), pp. 155-186.

RUFENACH, C. L., and ALPERS, W., 198I, Imaging ocean waves by synthetic aperture radars with long integration lines. I.E.E.E. Transactions on Antennas and Propagation, 29, 422-428.

Schroeter, J., Feindt, F., Alpers, W. R., and Keller, W. C., 1986, Measurements of the ocean wave-radar modulation transfer function at $4 \cdot 3 \mathrm{GHz}$. Journal of Geophysical Research, 91, 923-932.

Si:IChter, H., 1980, Two-dimensional power spectra of SEASAT-SAR imagery. Proceedings of the 3rd Seasat-SAR Workshop on SAR Image Quality, held in Frascati, Italy on 11-12 December 1980, ESA Publication SP-172 (Paris; European Space Agency), pp. $11-12$.

SwiFT, C. F., and WILSON, L. R., 1979, Synthetic aperture radar imaging of ocean waves I.E.E.E. Transactions on Antennas and Propagation, 27, 725-729.

TUCKer, M. J., 1983, The effect of a moving sea surface on SAR imagery. In Satellite Microwave Remote Sensing, edited by T.D. Allan (Chichester: Ellis Horwood Ltd.), pp. 146-154.

TUCKER, M. J., 1985, The decorrelation time of microwave echoes from the sea surface. International Journal of Remote Sensing, 6, 1075-1089.

Valenzuela, G. R., 1980, An asymptotic formulation for SAR images of the dynamical ocean surface. Radio Science, 15, 104-114.

VeSECKY, J. F., and StewaRT, R. H., 1982, The observation of ocean surface phenomena using imagery from the seasat synthetic aperture radar-an assessment. Journal of Geophysical Research, 87, 3397-3430.

Winebrenner, D. P., and Hasselmann, K., 1988, Specular point scattering contribution to the mean SAR image of the ocean surface. Journal of Geophysical Research, 93, 9281-9294. 
Wright. J. W., 1968, A new model for sea clutter. I.E.E.E. Transactions on Antennas and Propagation, 16, 217-223.

Wright, J. W., Plant W. J., Keller, W. C., and Jones, W. L., 1980, Ocean wave radar modulation transfer functions from the West Coast Experiment. Journal of Geophysical Research, 85, 4957-4966. 